Jülich, HLRZ 26/97

Bielefeld, ZIF-MS-10/97

Cambridge, MIT-CTP-2656

\title{
Chiral transition and monopole percolation in lattice scalar QED with quenched fermions
}

\author{
Wolfgang Franzki \\ Institut für Theoretische Physik E, RWTH Aachen, D-52056 Aachen, Germany \\ John B. Kogut ${ }^{1}$ \\ Center for Theoretical Physics, Massachusetts Institute of Technology, \\ 77 Massachusetts Avenue, Cambridge, MA 02139-4307 \\ Maria-Paola Lombardo \\ Zentrum für interdisziplinäre Forschung, \\ Universität Bielefeld, D-33615 Bielefeld, Germany
}

\begin{abstract}
We study the interplay between topological observables and chiral and Higgs transitions in lattice scalar QED with quenched fermions. Emphasis is put on the chiral transition line and magnetic monopole percolation at strong gauge coupling. We confirm that at infinite gauge coupling the chiral transition is described by mean field exponents. We find a rich and complicated behaviour at the endpoint of the Higgs transition line which hampers a satisfactory analysis of the chiral transition. We study in detail an intermediate coupling, where the data are consistent both with a trivial chiral transition clearly separated from monopole percolation and with a chiral transition coincident with monopole percolation, and characterized by the same critical exponent $\nu \simeq 0.65$. We discuss the relevance (or lack thereof) of these quenched results to our understanding of the $\chi U \phi_{4}$ model. We comment on the interplay of magnetic monopoles and fermion dynamics in more general contexts.
\end{abstract}

$\overline{1}$ On sabbatic leave from the University of Illinois at Urbana-Champaign

Preprint submitted to Elsevier Preprint

7 July 1997 


\section{Introduction}

Lattice gauge models with charged scalars and fermions have been studied both analytically and numerically for some time $[1,2]$. Their phase diagrams display a rich structure with chiral and Higgs transitions, as well as monopole condensation and percolation, and other topological excitations.

In this paper we study the interplay of chiral symmetry breaking and monopole percolation in scalar QED with quenched fermions. Abelian models can be rewritten by a duality transformation as theories of topological excitations, which help in determining their phase structure and critical behaviour [3]. A classic example is the confinement transition of the pure compact $U(1)$ lattice model, where monopole condensation was identified long ago as the underlying physical mechanism [4]. It is then interesting to investigate whether magnetic monopoles affect fermionic theories, and, in particular, the physics of chiral symmetry breaking and restoration. The work of [5] provides a theoretical framework for the study of the interplay of monopole and fermion dynamics, and a numerical strategy for its quantitative verification. Unfortunately, it has not been developed into a predictive theory, complete with equations coupling monopole and fermion observables and their interrelated scaling laws. Rather, it is just a 'physical picture' which could even be internally inconsistent. The speculative scenario is interesting and will be summarized here because we will find some support for it in our simulation data (although more conventional scenarios can accomodate the data as well).

Consider massless, pointlike fermions propagating in an ensemble of $U(1)$ monopoles. Suppose that the fermions experience only the $U(1)$ gauge interaction. The fermion-monopole interaction is purely topological in origin because its strength is given by the product of the electric charge $e$ and the magnetic charge $g$ which are related by the Dirac quantization condition, $e g=2 \pi$. This condition is renormalization group invariant, guarantees that the fermion-monopole interaction is always 'strong' and survives conventional perturbative screening, and lies at the heart of electric-magnetic duality. Suppose that the monopole network in the vacuum state of such an abelian $U(1)$ model experiences a second order phase transition at a certain coupling $e_{M}$ where the monopole loops grow without bound. Let $\nu$ denote the correlation length exponent for the transition. Suppose also that ordinary photon exchange is not strong enough to cause a chiral transition near $e_{M}$. Then, since the fermion's chirality is not a conserved property in the presence of the pointlike monopole, the monopole transition could induce a chiral transition and the two point correlation function of $\bar{\psi} \psi$ should inherit the critical exponent $\nu$ from the monopole-loop transition. Past measurements of monopole percolation have determined $\nu$ with good accuracy, $\nu \simeq 0.67$, which is near the correlation length exponent of ordinary bond percolation in four dimensions. 
In any case, since $\nu$ is distinct from the mean field value of $1 / 2$, this mechanism has been cited as a potential origin of interacting fermion field theories.

This mechanism has been explored so far in non-compact models [5-9], where, however, monopole solutions cannot be found analytically, monopoles are (naively) expected to decouple in the continuum limit, the Dirac string costs action and static monopoles do not generate long-range Coulomb fields. The ideal testing ground for the scenario of [5] is a simple model with compact fields, a second order chiral transition and monopole percolation. When we begun this study the $U(1)$ chiral transition was thought to be first order ${ }^{2}$. These considerations lead us to consider a fermion-scalar-compact $U(1)$ model, where the existence of a second order chiral transition was well established both analytically and numerically [14,15]. Note that this transition separates confinement from Higgs phases, while the QED chiral transition separats confining and Coulomb phases.

From a field theory point of view, this work continues the search for an interacting field theory which is strongly coupled at short distance [16]. Considerable work along this line has been invested in the study of the pure scalar-gauge model, with compact and non-compact gauge fields. Detailed numerical studies have supported the idea that non-compact scalar QED is trivial à la $\lambda \phi^{4}$ by finding evidence for the tell-tale logarithms $[17,18]$. Analogous results were reported for the compact model in [19]. The simulation results support the longheld theoretical prejudice that there is complete charge screening and the renormalized gauge coupling vanishes. In addition, in non-compact scalar QED monopole percolation turns out to be unrelated with the Higgs transition [18]. These results rise the question if the chiral nature of the transition in fermionic models might make the continuum limit non-trivial.

In a particle physics context, fermion-gauge-scalar models have been considered as possible toy models for the strongly coupled standard model $[20,21]$. More recently, one such lattice model - dubbed in this context $\chi U \phi_{4}$ - has been reconsidered in [22-27] as a new mechanism for spontaneous symmetry breaking and dynamical mass generation. Recent results showed that the chiral transition line flows into the Higgs transition line, terminating with a tricritical point, whose critical behaviour indicates nonpertubative renormalizability, consistent with a Yukawa theory. The long term aim is to find out whether and how the physics of the $\chi U \phi_{4}$ model is different from that of the usual Higgs-Yukawa mechanism of fermion mass generation. For this reason, the triviality issue is not really central here: it might well be that the model

2 The recent results on pure compact QED [10-12] indicate a second order transition and suggest that compact QED could be a very interesting testing ground for the scenario discussed in this paper, possibly also for the full theory, if negative $\gamma$ is chosen [13] (extended Wilson action with $-\gamma \sum_{P} \cos \left(2 \Theta_{p}\right)$ term). 
provides an alternative to the usual Higgs-Yukawa mechanism, with higher upper bounds for its validity.

It would be interesting if the results of this paper could be of use to further sharpen the understanding of the tricritical point of the $\chi U \phi_{4}$ model. However, this is a quenched study and its relationship with the full model can only be judged a posteriori: a better understanding of the $\chi U \phi_{4}$ model, and/or of the validity of the quenched approximation, is not the primary scope of this work. Our aim here is to illustrate some general dynamical mechanisms possibly relevant to continuum physics by investigating the interplay between chiral phase transition and monopole percolation. We choose to do so in the simplest possible situation: a scalar $U(1)$ gauge model with quenched fermions.

This paper is organized as follows: first we review the action and phase diagram of the $\chi U \phi_{4}$ model. We continue by discussing the topological excitations of Abelian models, and the observables relevant for the chiral transition. Section 4 gives an overview of the results for the topological excitations and show their correlations with other observables characterizing the phase diagram. These results confirm and extend the previous work of [28]. After this general study, we focus on three points along the chiral transition line: in Section 5 we present the results for the percolation of magnetic monopoles, in Section 6 those for the chiral transition. We conclude with a brief summary and discussion.

\section{Action and phase diagram}

The $\chi U \phi_{4}$ model is defined by the action [22]

$$
\begin{aligned}
S & =S_{\chi}+S_{U}+S_{\phi} \\
S_{\chi} & =\frac{1}{2} \sum_{x} \bar{\chi}_{x} \sum_{\mu=1}^{4} \eta_{x \mu}\left(U_{x, \mu} \chi_{x+\mu}-U_{x-\mu, \mu}^{\dagger} \chi_{x-\mu}\right)+a m_{0} \sum_{x} \bar{\chi}_{x} \chi_{x} \\
S_{U} & =-\beta \sum_{P} \cos \left(\Theta_{P}\right) \\
S_{\phi} & =-\kappa \sum_{x} \sum_{\mu=1}^{4}\left(\phi_{x}^{\dagger} U_{x, \mu} \phi_{x+\mu}+\text { h.c. }\right) .
\end{aligned}
$$

$\chi_{x}$ are the Kogut-Susskind fermion fields, $\phi_{x}$ is a scalar field with frozen length $\left|\phi_{x}\right|=1$, and $U_{x, \mu}$ represents the compact link variable. $U_{x, \mu \nu}$ is the plaquette product of the link variables $U_{x, \mu}$ and $\eta_{\mu x}=(-1)^{x_{1}+\cdots+x_{\mu-1}}$.

The phase diagram of the quenched approximation is shown in figure 1. It has two phases, the Coulomb phase and the confinement-Higgs phase. The second is separated into two regions with different realizations of chiral symmetry, 


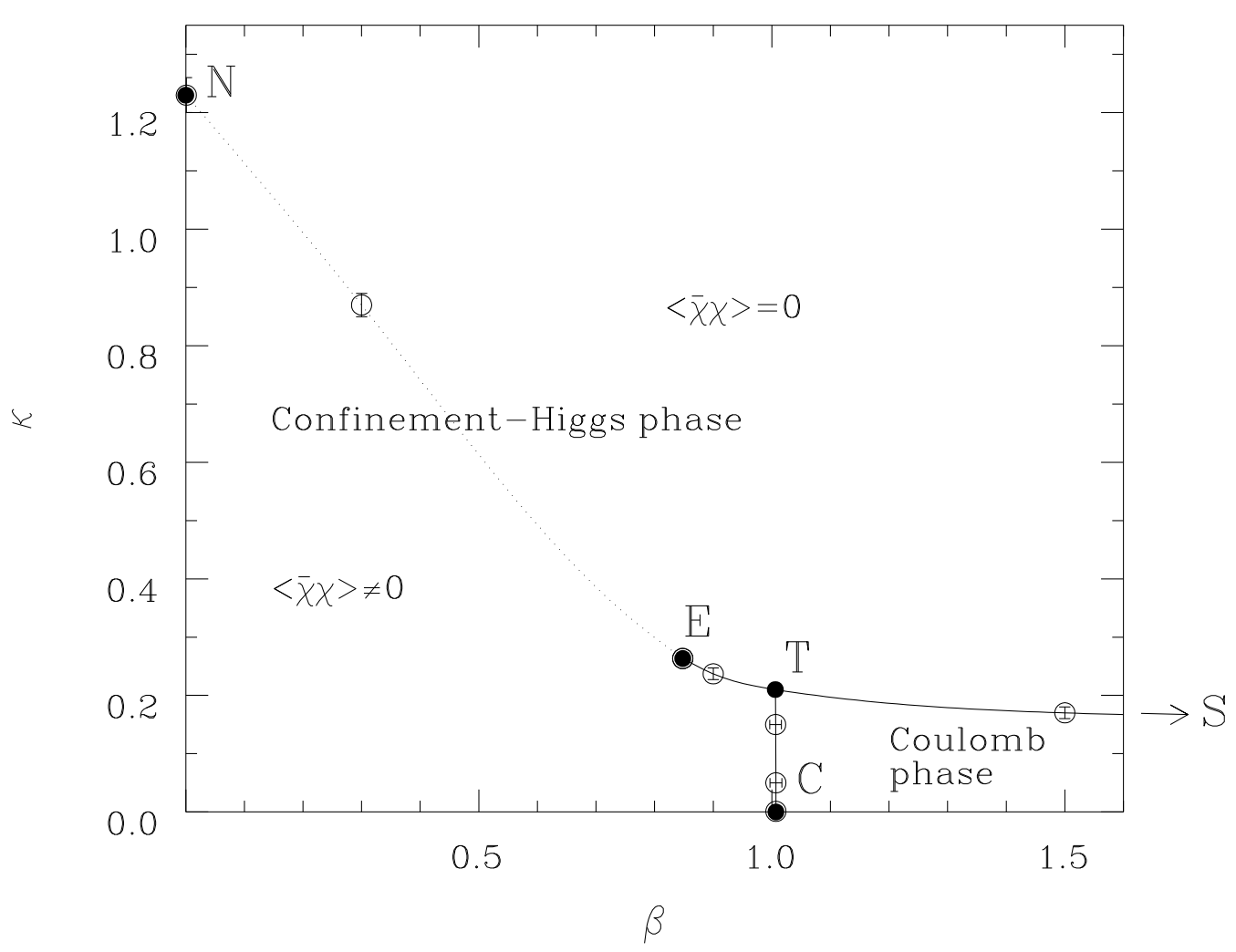

Fig. 1. The phase diagram of the Higgs model $\left(\chi U \phi_{4}\right.$ model for $a m_{0}=\infty$ in the update). The chiral transition of the quenched $\chi U \phi_{4}$ model is marked dotted. The points simulated in this work are those marked by open circles and the points $\mathrm{N}$ and E. The labels are the same as in $[22,23]$.

which are seperate phases in the chiral limit of the full model. The region with small $\kappa$ and $\beta$ is chirally asymmetric, the one with large $\beta$ and $\kappa$ is symmetric. These two regions are sometimes also called the confinement and Higgs regions, respectively, because of the limiting cases $\kappa=0$ and $\beta=\infty$.

For $\beta=0$ the gauge and scalar fields can be integrated out exactly, leading to a lattice version of the NJL model [14] (point N). The point E is the critical end point of the Higgs phase transition line ETC, which is a tricitical point in the chiral limit of the full model. $\mathrm{T}$ is the triple point and $\mathrm{C}$ the phase transition from the confinement (at strong gauge coupling) to the Coulomb phase (at weak gauge coupling) in the model without the scalar field. $\mathrm{S}$ is the critical point of the spin model $(\beta=\infty)$.

The status of the current investigation of the full model, including the dependence on the bare mass, can be found in [23-27]. Even though past lattice studies in the full model concentrated mostly on the tricritical point E, the scaling behavior along the chiral phase transition NE except $\mathrm{E}$ is believed to be NJL-like with reasonable accuracy [23]. At E the scaling behavior is different, e.g. the exponents seem to be nonclassical, which makes the point 
particularly interesting.

In this paper we study the quenched model, which corresponds to the limit $a m_{0}=\infty$ in the update. The dynamics is the same as in scalar QED, or Abelian Higgs model, and $S_{\chi}$ is only considered for the measurement part. The qualitative features of the phase diagram are known to be the same as in the full model. The endpoint of the Higgs transition line $\mathrm{E}$ is known with great accuracy [19]. We expect that also in the quenched model the point $\mathrm{N}$ is described by mean field exponents, as observed in [29], probably with logarithmic corrections. The topological excitations of the model have been considered in [28]. In our study we will characterize more fully the phase diagram using topological excitations and we will study in quantitative detail the chiral transition line extending from the point $\mathrm{N}$ to the endpoint $\mathrm{E}$, and its interplay with the monopole percolation.

\section{Operators}

In Abelian models with compact fields the periodic nature of the interactions makes it possible to rewrite the Action in terms of its topological excitations: magnetic monopole loops in pure compact QED, and closed vortex surfaces for pure scalar matter. For scalar-gauge systems there are strings of magnetic flux, i. e. lattice monopoles, closed vortex surfaces and open surfaces bounded by monopole loops. These topological excitations, investigated in the continuum in [30], are amenable to numerical studies on the lattice $[28,31,32]$. We review the main definitions below.

The study of the chiral transition will follow [33-35]. The relevant operators are reviewed in the last subsection.

\subsection{Monopoles}

For the measurement of the monopole loop density we introduce the flux variables [31]

$$
\theta_{\rho \sigma}(x)=\theta_{x, \rho}+\theta_{x+\rho, \sigma}-\theta_{x+\sigma, \rho}-\theta_{x, \sigma} \in(-4 \pi, 4 \pi]
$$

where $\theta_{x, \rho} \in(-\pi, \pi]$ is the phase angle of the link variables $U_{x, \rho}$ and the physical flux

$$
\bar{\theta}_{\rho \sigma}(x)=\theta_{\rho \sigma}(x)+2 \pi n_{\rho \sigma}(x) \in(-\pi, \pi]
$$


where $n_{\rho \sigma}(x)$ is an integer. If $n_{\rho \sigma}(x) \neq 0$ a Dirac sheet goes through the plaquette. The balance of the flux entering and leaving a 3-cube defines (on the dual lattice) a monopole current

$$
2 \pi m_{\mu}(x)=\varepsilon_{\mu \nu \rho \sigma} \nabla_{\nu} \bar{\theta}_{\rho \sigma}(x)
$$

The operator $\nabla_{\nu}$ labels lattice differentiation. The vector $m_{\mu}(x)$ is the total flux out of the cube $c$ at the dual site $x$ in direction $\mu$ :

$$
2 \pi m_{\mu}(x)=\sum_{p \in \partial c} \bar{\theta}_{p}=\sum_{p \in \partial c}\left(\theta_{p}+2 \pi n_{p}\right)=2 \pi \sum_{p \in \partial c} n_{p} .
$$

The loops of the monopoles (on the dual lattice) are closed, because of current conservation

$$
\nabla_{\mu} m_{\mu}(x)=0
$$

They are gauge invariant objects, whereas the Dirac sheets, apart from their boundaries, can be distorted.

From this we calculate the monopole loop density, in short monopole density, as

$$
\rho_{m}=\frac{1}{V}\left\langle\sum_{x, \mu}\left|m_{\mu}(x)\right|\right\rangle .
$$

Monopole percolation is detected using an order parameter borrowed from standard percolation models [36]. A connected cluster of monopoles is introduced [32]: one counts the number of dual sites $n$ which are connected with each other by monopole line elements. $n$ is the size of the cluster. Note that this construction ignores the vector structure of the monopole currents.

The density of the occupied bonds reads

$$
p_{m}=\frac{n_{\mathrm{tot}}}{4 V}=\frac{1}{4 V} \sum_{n=4}^{n_{\max }} g_{n} n
$$

where $g_{n}$ is the number of clusters with size $n, n_{\max }$ is the size of the largest cluster, and $n_{\text {tot }}$ is the total number of connected sites.

An order parameter for percolation is

$$
M_{\mathrm{perc}}=\frac{n_{\mathrm{max}}}{n_{\mathrm{tot}}}
$$


Its associated susceptibility is

$$
\chi_{M}=V\left(\left\langle M_{\text {perc }}^{2}\right\rangle-\left\langle M_{\text {perc }}\right\rangle^{2}\right) .
$$

We have also considered the same susceptibility as in [32]

$$
\chi_{\mathrm{perc}}=\left\langle\left[\sum_{n=4}^{n_{\max }} g_{n} n^{2}-n_{\max }^{2}\right] / n_{\mathrm{tot}}\right\rangle .
$$

\subsection{Vortex sheets}

For the measurement of the vortex sheets we introduce $[3,28]$

$$
\bar{\vartheta}_{\nu}(x)=\varphi_{x}-\theta_{x, \nu}-\varphi_{x+\nu}+a_{\nu}(x) \in(-\pi, \pi]
$$

where $\varphi_{x} \in(-\pi, \pi]$ is the phase angle of $\phi_{x}$ and $a_{\nu}(x)$ is a integer. The local vortex sheet is now

$$
V_{\rho \sigma}(x)=\varepsilon_{\rho \sigma \mu \nu}\left(\nabla_{\mu} a_{\nu}(x)+\frac{1}{2} n_{\mu \nu}(x)\right) .
$$

From this we calculate the vortex density as

$$
\rho_{V}=\frac{1}{6 V}\left\langle\sum_{x, \rho \sigma}\left|V_{\rho \sigma}(x)\right|\right\rangle .
$$

\subsection{Chiral condensate}

The chiral condensate is measured in the usual way

$$
\langle\bar{\chi} \chi\rangle=\left\langle\operatorname{Tr} M^{-1}\right\rangle
$$

with a stochastic estimator, where $M$ is the fermion matrix.

We also measured the logarithmic derivative of the chiral condensate [34]:

$$
R_{\pi}=\left.\frac{\partial \ln \langle\bar{\chi} \chi\rangle}{\partial \ln a m_{0}}\right|_{\beta, \kappa}=\left.\frac{a m_{0}}{\langle\bar{\chi} \chi\rangle} \frac{\partial\langle\bar{\chi} \chi\rangle}{\partial a m_{0}}\right|_{\beta, \kappa} .
$$


This can be expressed as ratio of zero momentum meson propagators $C(p=0)$ (susceptibilities):

$$
\left.\frac{\partial\langle\bar{\chi} \chi\rangle}{\partial a m_{0}}\right|_{\beta, \kappa}=C_{\sigma}(p=0), \quad \frac{\langle\bar{\chi} \chi\rangle}{a m_{0}}=C_{\pi}(p=0)
$$

where the second is the Ward identity, which results from the chiral $U(1)_{A}$ symmetry of the staggered fermions [37]. This gives

$$
R_{\pi}=\frac{C_{\sigma}(p=0)}{C_{\pi}(p=0)}
$$

We note that in the quenched approximation only the connected part of $C_{\sigma}$ need to be considered, since fermion loops are neglected [38]. We have checked that our measurement of $R_{\pi}$ using eq. (17) is in good agreement with the logarithmic derivative of the chiral condensate computed by numerical differentiation of the $\langle\bar{\chi} \chi\rangle$ results.

\section{Measurement of the topological excitations - Overview}

Fig. 2 shows a overview of the results on the $6^{4}$ lattice. For $\beta=0.00$ (a) (NJL line) all observables are very smooth, only the ratio $M_{\text {perc }}$ and the chiral condensate show a somewhat steeper descent. Monopole and vortex density decrease slowly for increasing $\beta$. More to the right on the NE line for $\beta=$ 0.60 (b) a similar behavior is observed but the transition region is somewhat smaller.

For $\beta=0.90$ (c) (crosswise to the ET line first order) a steep descent in all observables at $\kappa=0.24(1)$ can be observed, as this is typical for first order phase transitions. Obviously the monopoles and the vortices are sensitive to this phase transition.

At $\beta=1.50$ (d) (crosswise to the TS line first order) a phase transition from Coulomb to Higgs phase can be observed. At this phase transition only the vortex density and the link energy are sensitive, which vary rapidly at $\kappa=0.17$. The other observables stay nearly constant.

The phase transition CT from confinement to Coulomb phase is shown for $\kappa=0.05$ (e). At the phase transition at $\beta=1.01(1)$ the monopole density, $\rho_{m}$ and the chiral condensate steeply decrease. Link energy and vortex density are insensitive to this phase transition. 

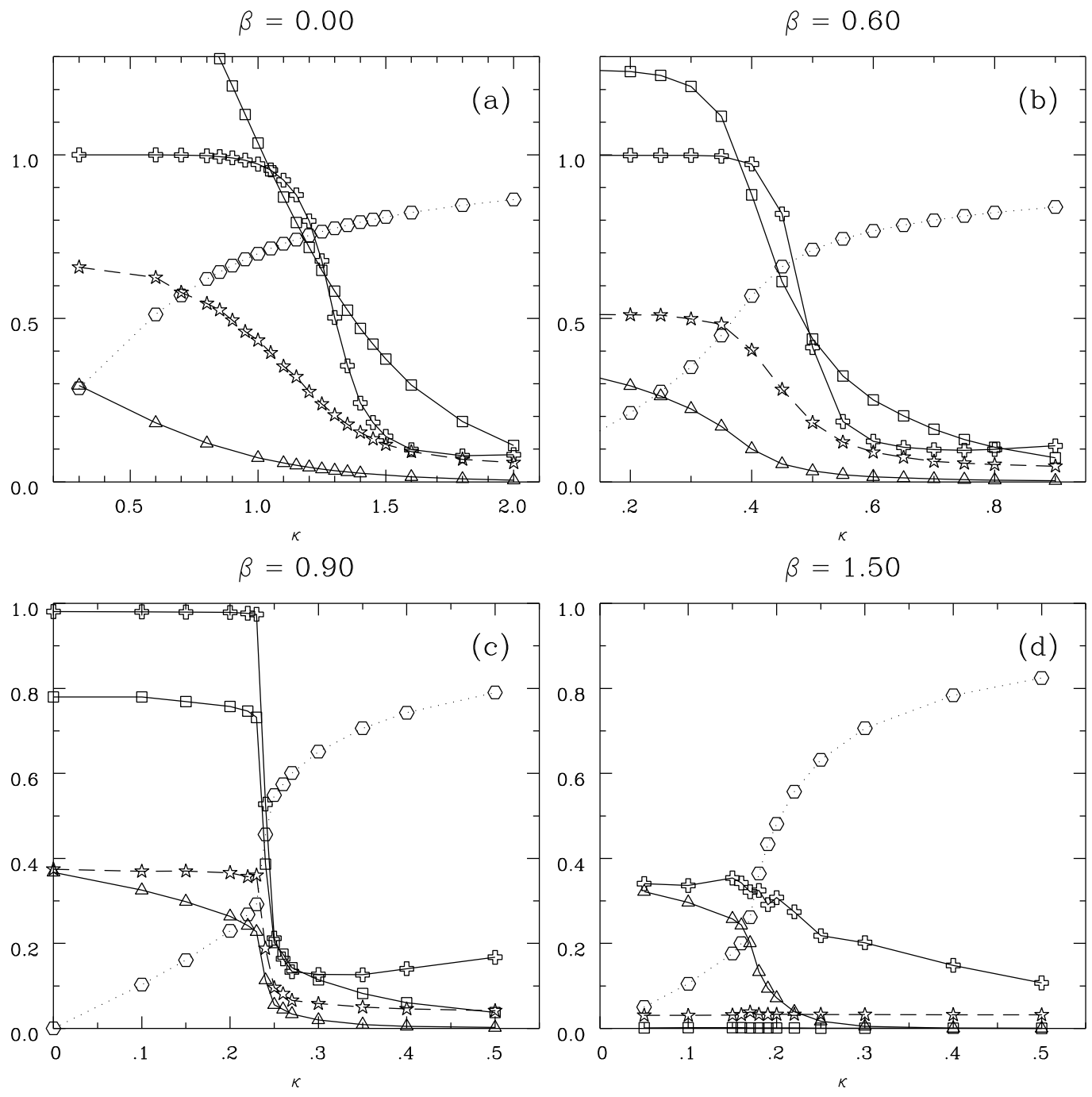

$\beta=1.50$

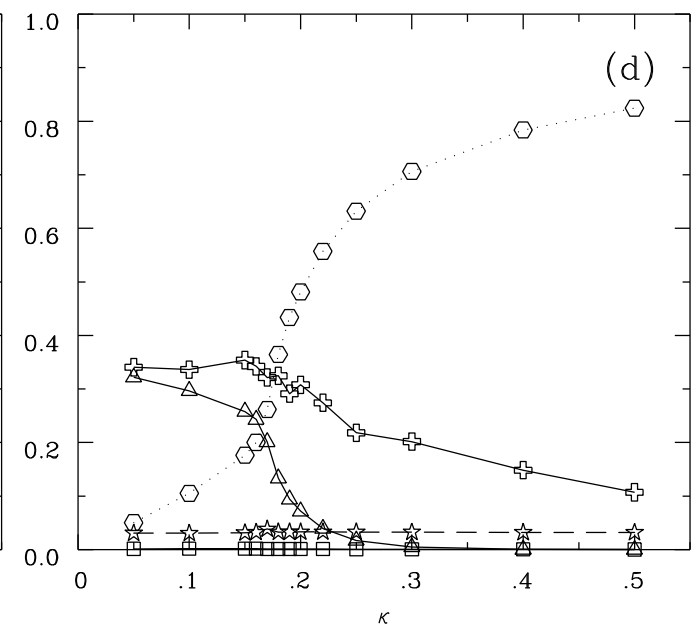

$\kappa=0.05$

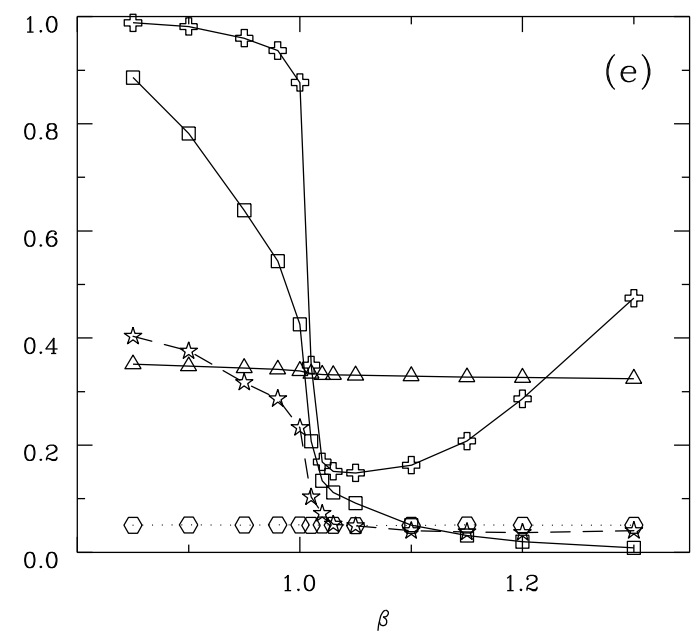

$$
\begin{aligned}
& \square: \rho_{\mathrm{m}} \\
& \rightsquigarrow: \mathrm{M}_{\text {perc }}=\mathrm{n}_{\text {max }} / \mathrm{n}_{\text {tot }} \\
& \triangle: \rho_{\mathrm{V}} \\
& \square: \mathrm{E}_{\mathrm{L}} \\
& \text { « }:\langle\bar{X} X\rangle
\end{aligned}
$$

Fig. 2. Overview of the topological observables $\rho_{m}, M_{\text {perc }}$ and $\rho_{V}$ together with $E_{L}$ and $\langle\bar{\chi} \chi\rangle$ on the $6^{4}$ lattice at the lines NE $(\mathrm{a}+\mathrm{b})$, ET (c), TS (d) and CT (e). The errors (not shown) are nearly always smaller than the symbols. 

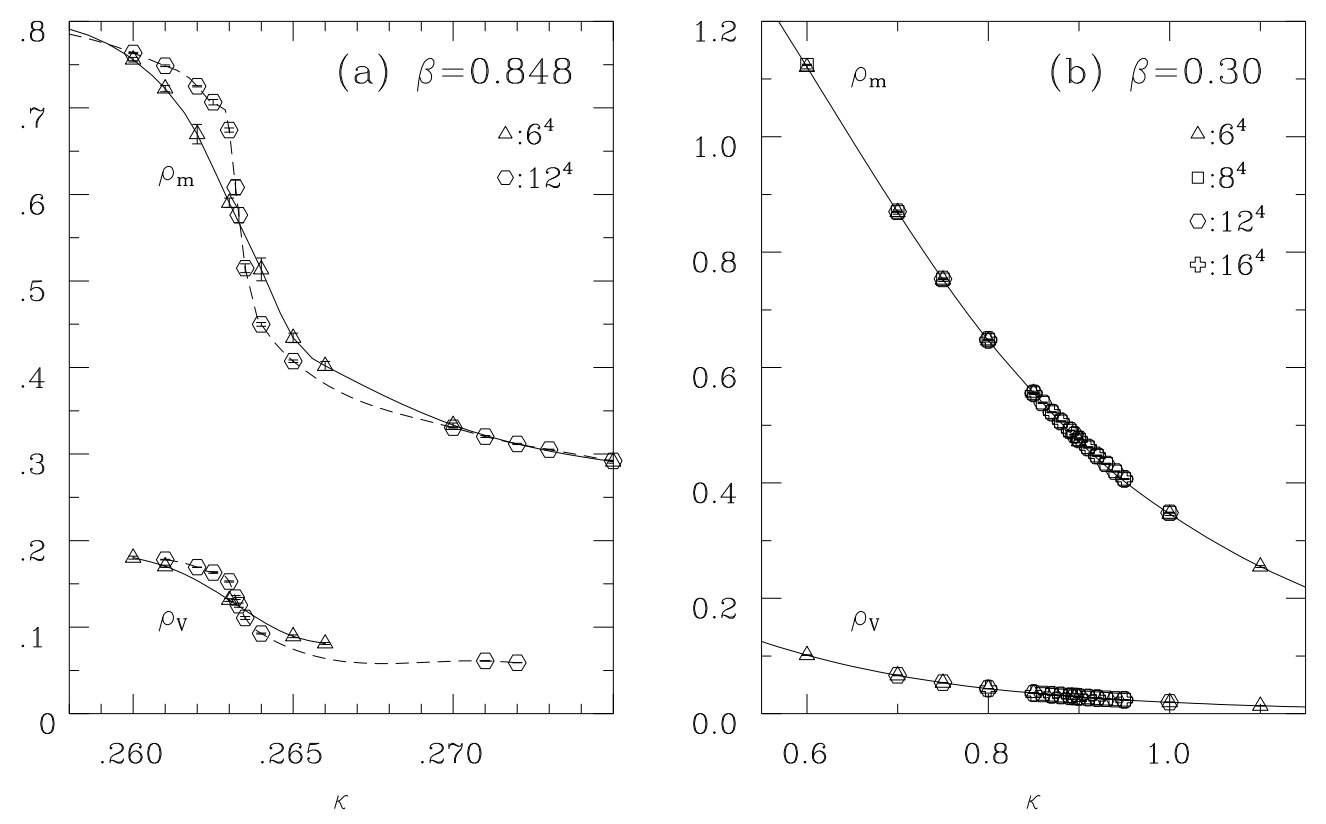

Fig. 3. Monopole density $\rho_{m}$ and vortex density $\rho_{V}$ for (a) $\beta=0.848 \simeq \beta_{\mathrm{E}}$ and (b) $\beta=0.30$.

So, we have the following picture. Consider the three regions of the phase diagram: Coulomb phase, Higgs region and confinement region. In the confinement region the monopole and vortex density are large and in the Higgs region both are small. In the Coulomb phase the vortex density is large and the monopole density small. At the first order phase transtions the observables which are large in one phase and small in the other show a steep decrease, whereas the others are insensitive.

The order parameter of percolation $M_{\text {perc }}$ follows (essentially) the monopole density but with more rapid changes. This could be understood in analogy to bond percolation. If the monopole density is high, percolating clusters are very likely, whereas for small monopole densities they are very unlikely. The first percolating clusters occur at some intermediate coupling. For large $\beta$, along the line of first order phase transitions, this seems to coincide with the phase transition of the monopole density. Such interplay of monopole condensation and percolation has been investigated before in pure compact QED in [39].

At the line NE, which is second order in the full theory, the situation is less obvious.

At the endpoint $\mathrm{E}$ of the Higgs phase transition, $\beta=0.848, \rho_{m}$ and $\rho_{V}$ show a steep descent, which gets increasingly steeper for increasing lattice size (Fig. 3a). The derivative of these observables show an increasing maximum. We have not done a scaling investigation but the data suggest a divergence.

For $\beta=0.30$ (Fig. 3b), still along the line NE, both densities show no max- 


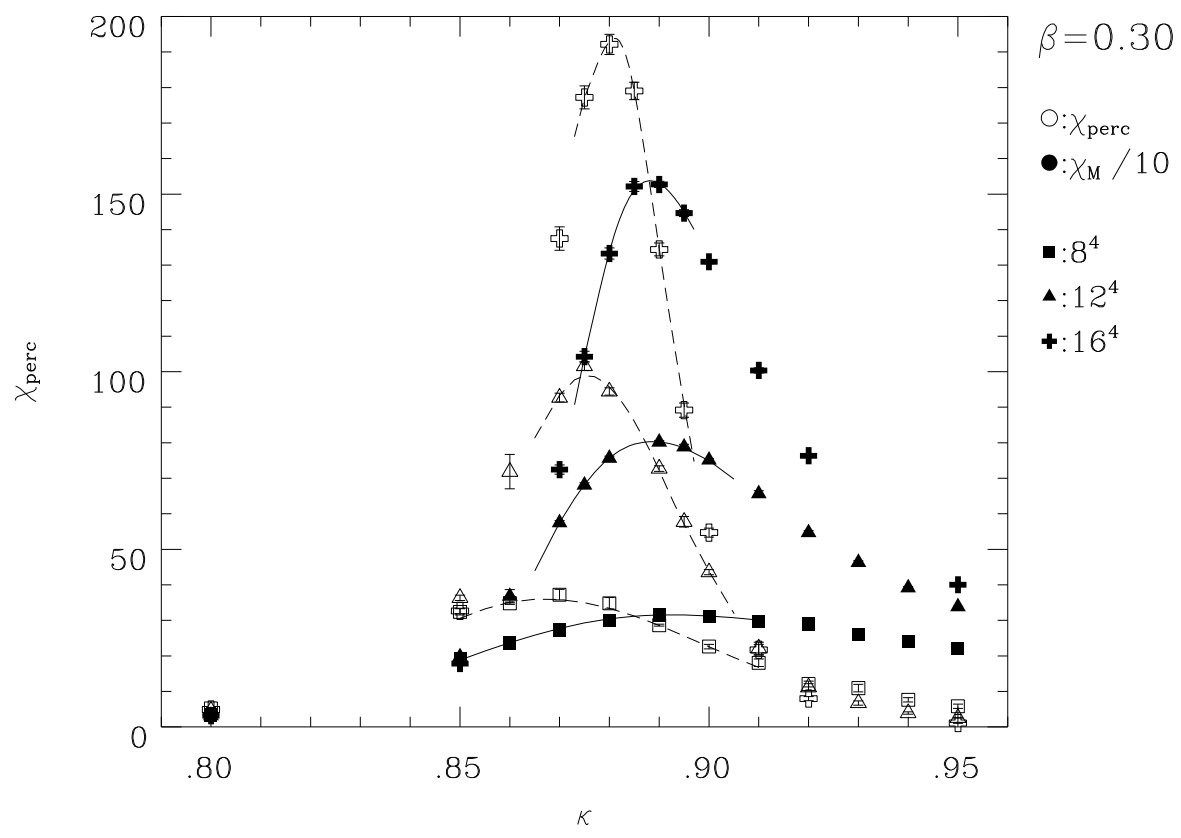

Fig. 4. Percolation susceptibilities $\chi_{\text {perc }}$ (solid) and $\chi_{M} / 10$ (open symbols) for $\beta=0.30$. The curves are interpolations made with the use of reweighting techniques.

imum in the derivative. Moreover, no volume dependence was observed. Obviously they do not get critical in the region where the chiral condensate has its largest variation.

For $\beta=0.00$ the picture is very similar to that at $\beta=0.30$.

The three $\beta$ values $0.00,0.30$, and 0.848 have been selected for the systematic investigation which we describe in the rest of this paper.

\section{Magnetic Monopoles in the vicinity of the NE line}

In this Section we measure the critical line for the percolation of the magnetic monopole clusters, and its critical exponents. An accurate measurement of the critical line is obtained thanks to the combined use of reweighting techniques, and the two definitions of monopole percolation $\chi_{\text {perc }}$ and $\chi_{M}$.

The quality of our measurements is demonstrated in Fig. 4 where we contrast $\chi_{\text {perc }}$ and $\chi_{M}$ at $\beta=0.30$ on lattices of different size. Both susceptibilities show a clear maximum for all $\beta$ 's in the vicinity of the line NE. The peaks are very close, and the volume corrections have different signs.

We have determined the precise position of the maximum of $\chi_{\text {perc }}$ and $\chi_{M}$ 
Table 1

Position and height of the maximum of the percolation susceptibility for different $\beta$ and lattice sizes along the line NE. The values are given for both definitions $\chi_{M}$ and $\chi_{\text {perc }}$. For the infinite volume extrapolation of $\kappa_{\max }(\kappa=1.3469, \kappa=0.8875$, and $\kappa=0.2714$, respectively) also the density of the occupied bonds $p_{m}$ and the monopole density $\rho_{m}$ is printed. For comparison, our best estimate for the chiral transition in the infinite volume is given in the second column (see section 6).

\begin{tabular}{|c|c|c|c|c|c|c|c|c|}
\hline$\beta$ & $\kappa_{\text {chiral }, c}$ & lattice & $\kappa_{\max }(L)$ & $\chi_{M}(L)$ & $\kappa_{\max }(L)$ & $\chi_{\text {perc }}(L)$ & $p_{m}$ & $\rho_{m}$ \\
\hline \multirow{5}{*}{0.00} & \multirow{5}{*}{$1.23(3)$} & $6^{4}$ & $1.307(4)$ & 156.(3) & $1.363(5)$ & $16.11(5)$ & $0.1132(1)$ & $0.5283(6)$ \\
\hline & & $8^{4}$ & $1.3179(6)$ & 342.(2) & $1.3525(6)$ & $32.00(4)$ & $0.11323(2)$ & $0.52852(9)$ \\
\hline & & $12^{4}$ & $1.3303(5)$ & 941.(9) & $1.3474(4)$ & $80.9(4)$ & $0.11322(1)$ & $0.52848(5)$ \\
\hline & & $16^{4}$ & $1.3358(6)$ & 1865.(13) & $1.347(1)$ & 154.(7) & $0.11322(1)$ & $0.52847(7)$ \\
\hline & & $20^{4}$ & $1.3395(3)$ & 3054.(31) & $1.3467(4)$ & 253.(3) & $0.11321(1)$ & $0.52841(4)$ \\
\hline \multirow{4}{*}{0.30} & \multirow{4}{*}{$0.87(2)$} & $6^{4}$ & $0.861(4)$ & $165 .(3)$ & $0.903(3)$ & $15.6(2)$ & $0.1074(3)$ & $0.494(1)$ \\
\hline & & $8^{4}$ & $0.8671(5)$ & 360.(2) & $0.8926(8)$ & $31.5(1)$ & $0.10746(3)$ & $0.4946(2)$ \\
\hline & & $12^{4}$ & $0.8756(4)$ & 989.(5) & $0.8886(8)$ & $80.3(4)$ & $0.10749(2)$ & $0.49474(9)$ \\
\hline & & $16^{4}$ & $0.8812(4)$ & 1937.(13) & $0.8882(5)$ & $153.7(7)$ & $0.10750(1)$ & $0.49477(7)$ \\
\hline \multirow{4}{*}{0.848} & \multirow{4}{*}{$0.26: 0.29$} & $8^{4}$ & $0.2678(1)$ & 574.(4) & $0.2732(1)$ & $22.35(6)$ & $0.0727(2)$ & $0.3180(7)$ \\
\hline & & $12^{4}$ & $0.2692(1)$ & 1437.(15) & $0.27204(7)$ & $59.5(3)$ & $0.07257(5)$ & $0.3174(2)$ \\
\hline & & $16^{4}$ & $0.2701(1)$ & 2758.(34) & $0.27191(8)$ & 120.(1) & $0.07259(7)$ & $0.3175(3)$ \\
\hline & & $20^{4}$ & $0.27065(5)$ & 4501.(53) & $0.27168(4)$ & $206.1(8)$ & $0.7257(2)$ & $0.3174(1)$ \\
\hline
\end{tabular}

with the multi-histogram method. The numerical results for $\chi_{\text {perc }}$ and $\chi_{M}$, together with other magnetic observables, are summarized in table 1. In Fig. 5 we show the position of the peak of the two susceptibilities as a function of $1 / L$ for $\beta=0.00$. We superimpose a power law fit with different amplitudes and exponents and a common critical point. Again we see that, by increasing volume, $\chi_{\text {perc }}$ approaches the critical point from above, $\chi_{M}$ from below. They provide upper and lower bounds, hence an accurate determination, of the critical coupling for the percolation of magnetic monopole clusters.

A determination of the critical exponent of comparable accuracy requires a careful consideration of scaling violating effects, and will be presented elsewhere. For our present purposes a precision comparable to that we achieved at the chiral transition would suffice $(5-10 \%)$.

Fig. 6 shows the scaling behavior of the maximum of $\chi_{\text {perc }}$ with the lattice size. Note the linear rise with size in this double logarithmic plot, supporting power law scaling. Only the data on the $6^{4}$ lattice displays small deviations. In a fit with the finite size scaling equation

$$
\chi_{\text {perc }, \max }(L) \propto L^{\gamma_{\text {perc }} / \nu_{\text {perc }}}
$$

we measure for $\beta=0.00$ and $\beta=0.30$ the nearly identical value of $\gamma_{\text {perc }} / \nu_{\text {perc }}=$ 2.28(2). The subscript 'perc' is added to indicate the definition of the exponents at the percolation transition. For comparison, the measurements in quenched non-compact [6] and in full non-compact QED [5] yields quite similar values of $\gamma_{\text {perc }} / \nu_{\text {perc }}=2.24(2)$ and $\gamma_{\text {perc }} / \nu_{\text {perc }}=2.25(3)$, respectively. For $\beta=0.848$ one obtains a somewhat larger value of $\gamma_{\text {perc }} / \nu_{\text {perc }}=2.42(1)$. 


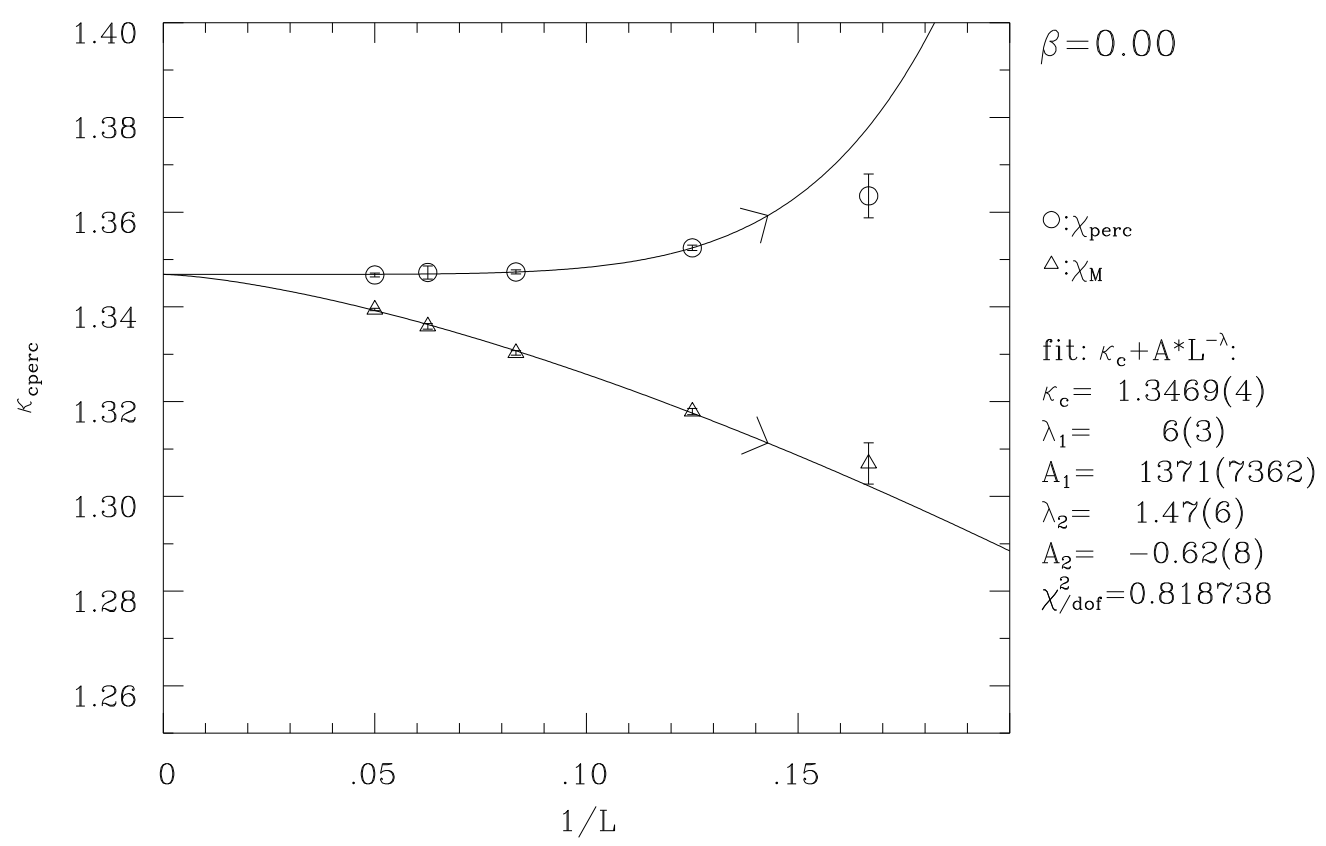

Fig. 5. Position of the peak of the percolation susceptibility $\chi_{\text {perc }}$ (circles) and $\chi_{M}$ (triangles) for $\beta=0.00$.

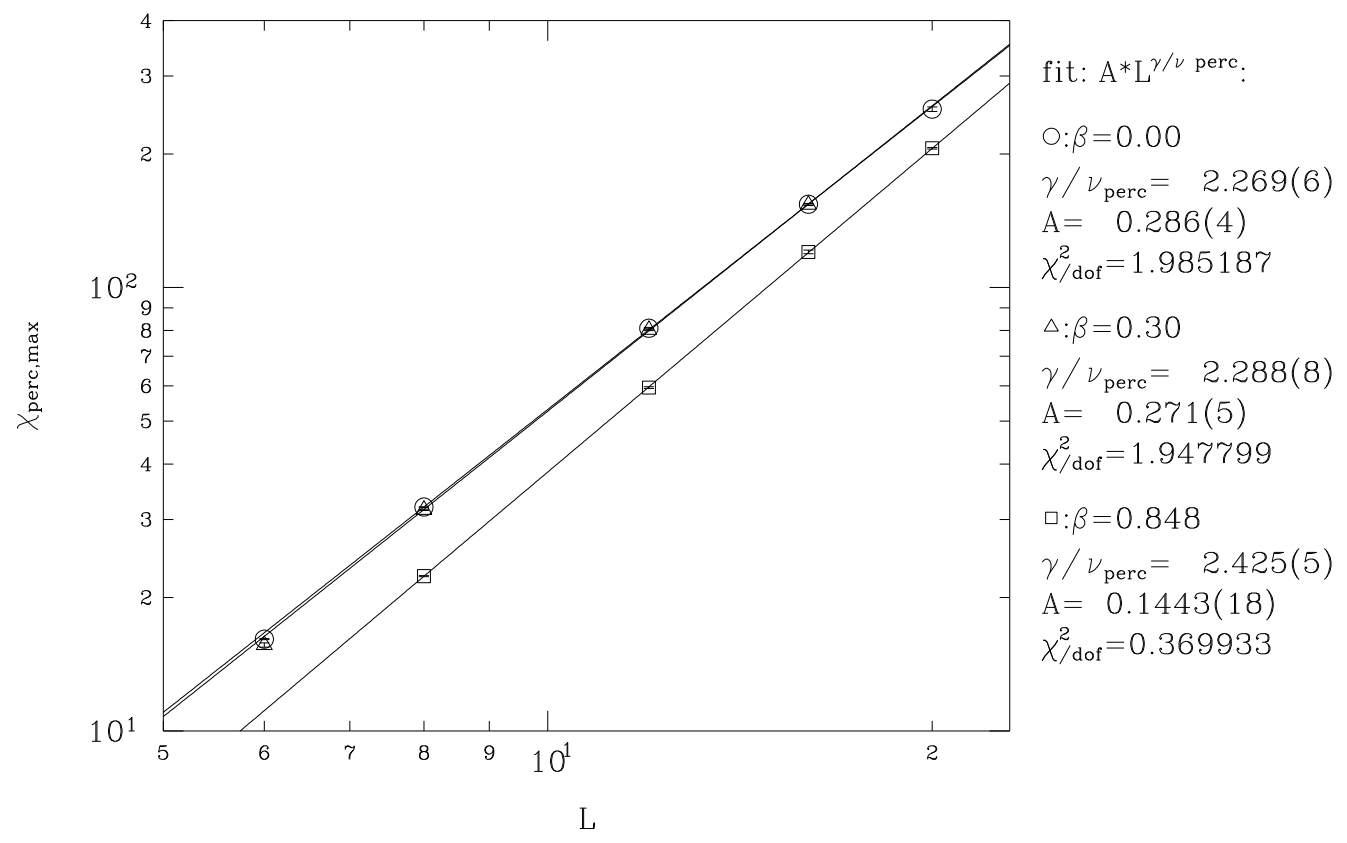

Fig. 6. Scaling behavior of the maximum of the percolation susceptibility $\chi_{\text {perc }}$ for $\beta=0.00, \beta=0.30$ and $\beta=0.848$. In the fit only data with $L \geq 8$ have been considered. 
The maximum of $\chi_{M}$ shows larger deviations from the leading finite size scaling behaviour for small lattices. If we use only lattices with $L \geq 12$, we get for $\beta=0.00$ and $\beta=0.30$ an exponent $\gamma_{M} / \nu_{M}=2.32(3)$ and for $\beta=0.848$ $\gamma_{M} / \nu_{M}=2.23(4)$. The subscript $M$ indicates the exponents which have been determined using $\chi_{M}$.

The $\gamma / \nu$ 's from the two definitions are separated by less than two standard deviations at $\beta=0.00$ and $\beta=0.30$. At $\beta=.848$ the disagreement increases. Larger lattices, and/or a careful consideration of correction to scaling terms should bring the results from the two definitions closer. Within this uncertainty all numbers of $\gamma / \nu$ of the percolation transition are compatible with 2.3(1).

We can contrast these results with those of pure random bond percolation [40]. For pure random bond percolation the critical density is $p_{c}=0.161$. In our model the density $p_{m, c}$ at the percolation phase transition depends on $\beta$, and it is generally lower. Our result is close to that of site bond percolation $\gamma / \nu=2.094(2)[41,40]$. Considering the vector character of the monopole current, we should not expect that the two models are in the same universality class, as pointed out in [6]).

To determine $\beta_{\text {perc }} / \nu_{\text {perc }}$ we investigated the scaling with volume of $M_{\text {perc }}$ at the (infinite volume) critical coupling. The results are very sensitive to the precise value of $\kappa_{c}$. We estimated anyway $\beta_{\text {perc }} / \nu_{\text {perc }}=0.85(3)$ in good agreement with the scaling relation

$$
\frac{\beta_{\text {perc }}}{\nu_{\text {perc }}}=\frac{1}{2}\left(d-\frac{\gamma_{\text {perc }}}{\nu_{\text {perc }}}\right) .
$$

The result for quenched non-compact $\mathrm{QED}$ is $\beta_{\text {perc }} / \nu_{\text {perc }}=0.88(2)$.

The exponent $\beta_{\text {perc }}$ was determined from $M_{\text {perc }}$ in the phase with percolation, using the results not distorted by finite size effects. The result for $\beta=0.00$ is shown in figure 7 . Here again we fixed the critical coupling. The fits give $\beta_{\text {perc }}=$ $0.52(2)$, where the error was determined by varying the critical coupling in the interval $\kappa_{c}=1.346 \ldots 1.348$. We repeated this for $\beta=0.30$ and obtained a $\beta_{\text {perc }}=0.50(4)$, smaller but compatible within errors. $(\beta=0.58(2)$ in noncompact quenched QED [6].)

Together with our determination of $\beta_{\text {perc }} / \nu_{\text {perc }}=0.85(3)$ we get the exponent for the correlation length $\nu_{\text {perc }}=0.61(4)$ to be contrasted with that of non-compact QED $\nu_{\text {perc }}=0.66(3)$, and of pure random site percolation $\nu_{\text {perc }}=0.69(1)$. A more careful analysis, considering corrections to scaling, would be necessary to assess the significance of these differences and to decide if percolation is in the same universality class for all $\beta \leq 0.848$. Differences, if any, are anyway small. 


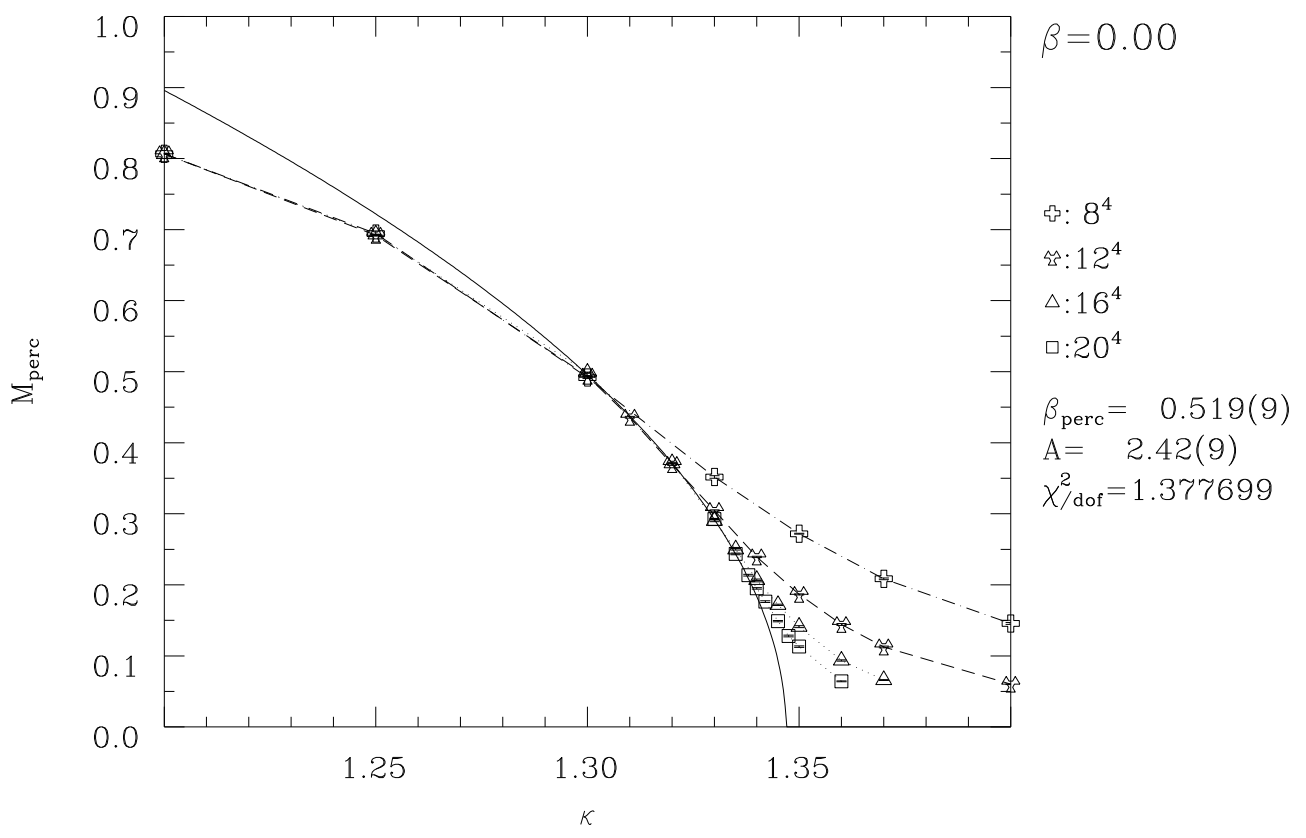

Fig. 7. Scaling behavior of $M_{\text {perc }}$ for $\beta=0.00$. In the fit only data with $L \geq 16$ in the interval $\kappa=1.30 \ldots 1.33$ have been considered. The critical coupling was fixed to $\kappa_{c}=1.3469$.

Note that for $\beta_{\mathrm{E}}=0.848$ the percolation transition at $\kappa=0.2714(3)$ is distinct from the Higgs phase transitions which occurs at $\kappa_{c}=0.26333(1)[19,25,26]$. In Fig. 8, we see that all the observables are very sensitive to the Higgs phase transition (note the sharp peak of the susceptibility of the link energy), with the exception of the susceptibility $\chi_{\text {perc }}$ of the percolation. This shows that at the endpoint, the Higgs phase transition and the percolation of the monopoles is uncorrelated. So the percolation of the monopoles probably can not help us understand the tricritical point in the dynamical model.

\section{The chiral transition (NE) line}

We consider first the behavior of the susceptibility ratio. The discussion here aims at illustrating the general trend along the critical line. In the second subsection we cross check the results by fitting the data to the equations of state. That includes a more detailed discussions on the selection of the scaling window, and a careful error analysis. 


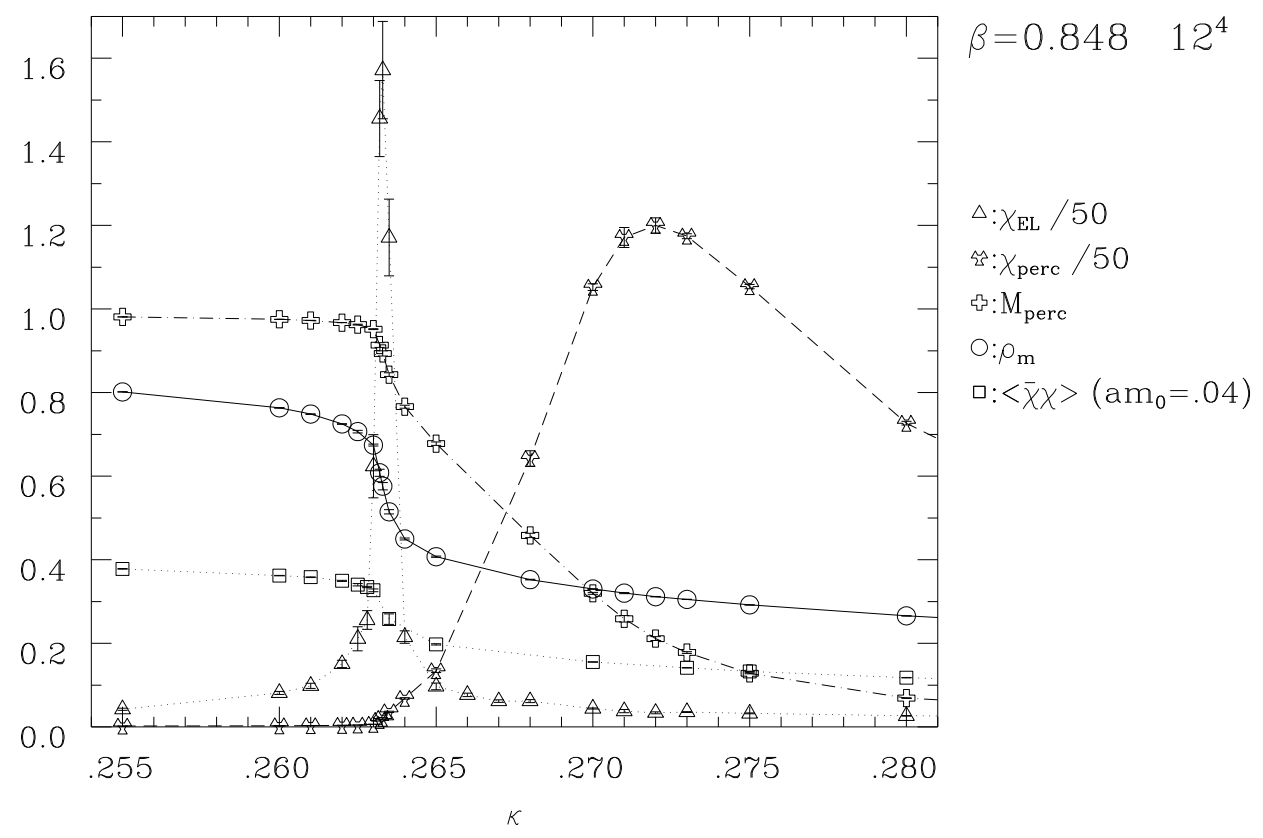

Fig. 8. Susceptibility of the monopole percolation $\chi_{\text {perc }}$ and the link energy $\chi_{E_{L}}$, as well as the order parameter of the percolation $M_{\text {perc }}$, the monopole density $\rho_{m}$ and the chiral condensate $\langle\bar{\chi} \chi\rangle\left(a m_{0}=0.04\right)$ as function of $\kappa$ on a $12^{4}$ lattice at $\beta=0.848$ at the endpoint of the Higgs phase transitions.

\section{$6.1 R_{\pi}$}

We measure the ratio of $R_{\pi}$ as a function of $a m_{0}$ : at the chiral transition $R_{\pi}$ should be independent on $a m_{0}$ if the transition is described by a simple scaling law. Logarithmic corrections modify this behaviour.

For $\beta=0.00$ (Fig. 9a) our data indicate a chiral transition at $\kappa_{c}=1.23(3)$ with $R_{\pi}=0.35(5)$. This value compares well with the expected mean field result $R_{\pi}\left(\kappa_{c}\right)=1 / \delta=1 / 3$. Logarithmic corrections, if any, are small, as we will see later on.

For $\beta=0.30$ (Fig. 9b) the results suggest $\kappa_{c}=0.87(2)$ with $R_{\pi}\left(\kappa_{c}\right)=0.45(10)$. This corresponds to $\delta=2.2(6)$. Logarithmic corrections à la Nambu-Jona Lasinio would predict

$$
R_{\pi}\left(\kappa_{c}\right)=1 /(3+1 / \log (\langle\bar{\chi} \chi\rangle))
$$

resulting in a slight upward trend of the critical ratio, still compatible with the data. We comment more on this point in the next subsection. The curves for $a m_{0}>.02-.04$ starts bending downwards, after the initial rise: masses larger than .04 cannot be used safely to assess the critical behaviour. In the next subsection we discuss in detail how to select the appropriate scaling window. 
(a)

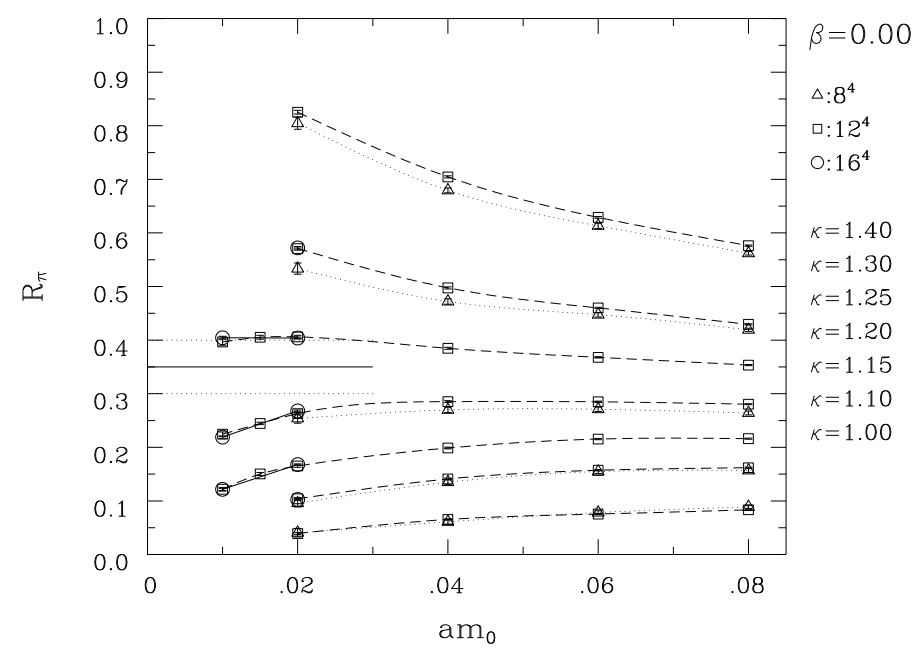

(b)

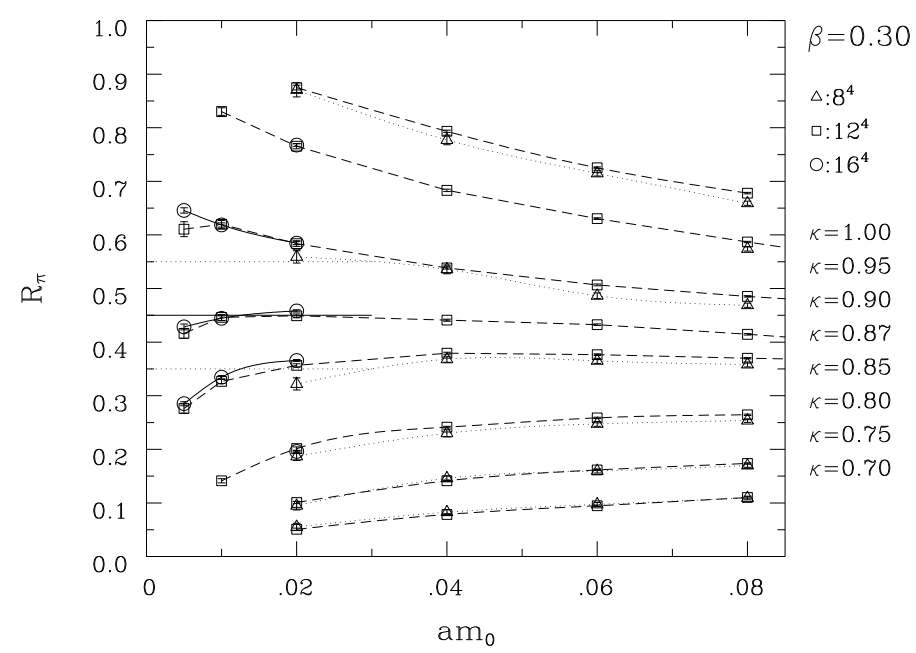

(c)

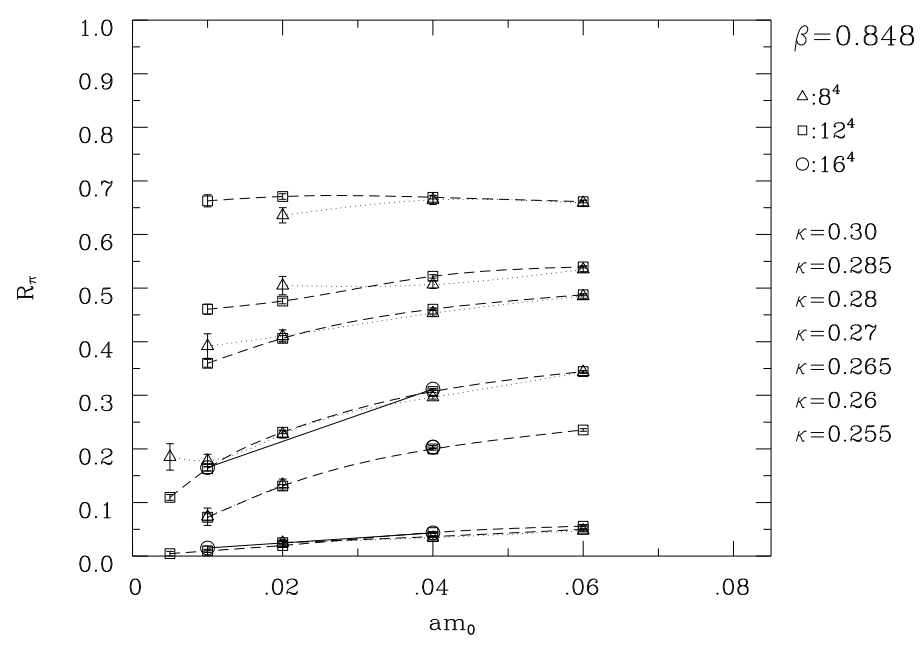

Fig. 9. $R_{\pi}$ as function of $a m_{0}$ at (a) $\beta=0.00$, (b) $\beta=0.30$ and (c) $\beta=0.848 \simeq \beta_{E}$. (Solid, dashed, dotted lines) are for $\left(16^{4}, 12^{4}, 8^{4}\right)$ lattices. Approximate values for the critical point are indicated with horizontal lines. 


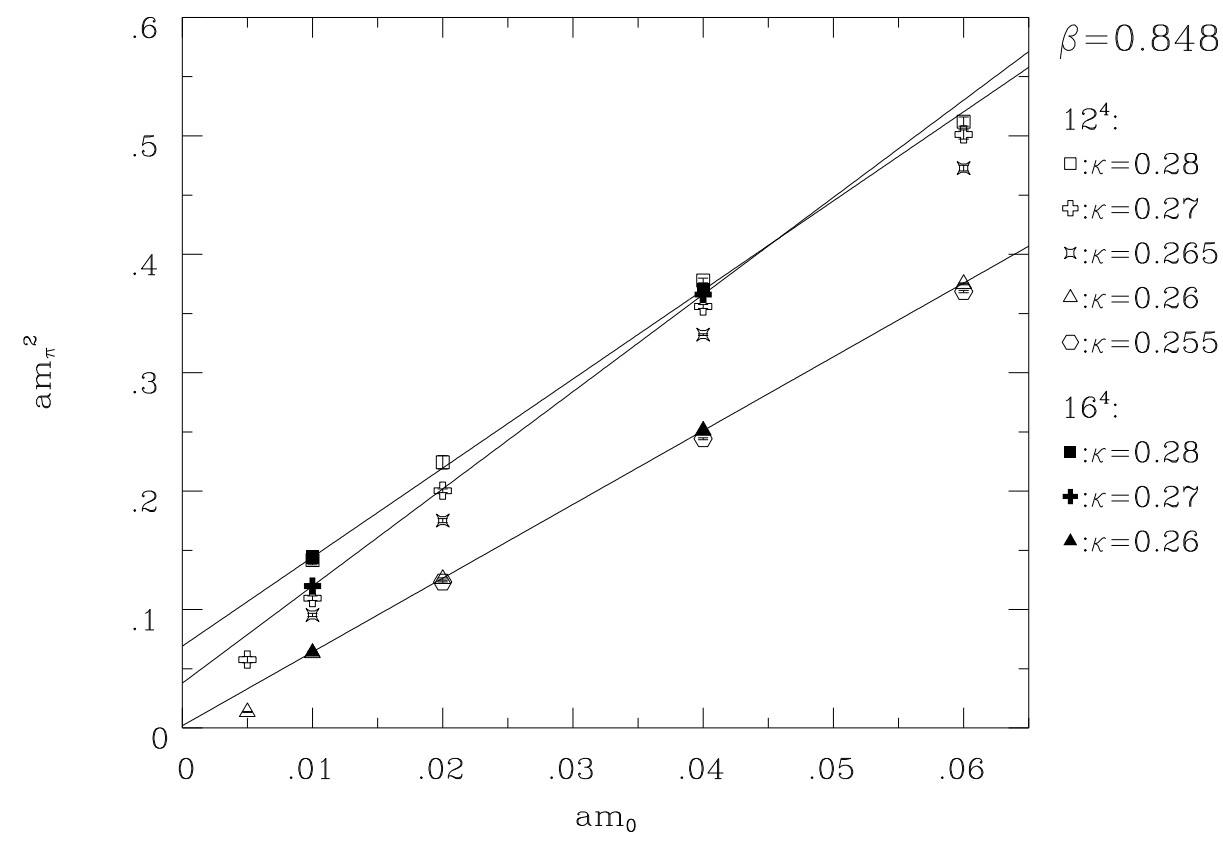

Fig. 10. Behavior of the quadratic $\pi$ meson mass as function of $a m_{0}$. In the broken phase a straight line through the origin is expected. The lines indicate a fit to the $16^{4}$ data. For $\kappa=0.27$ we observed large finite size effects, which make the extrapolation unreliable.

For $\beta=0.848$ (point $\mathrm{E}$ ) the Higgs phase transition is at $\kappa_{c} \simeq 0.263$. We have shown above that the Higgs transition is separate from monopole percolation, and here (Fig. 9c) we see that at this $\kappa$ a conventional second order chiral transition is incompatible with the data. The data in Fig. 9c would rather suggest a chiral transition in the interval [.28 - .30], even on the right of the percolation transition. But this behaviour is far from clear.

As an alternative strategy to locate the chiral transition at $\beta=0.848$ we investigated the scaling behavior of $\left(a m_{\pi}\right)^{2}$ as a function of $a m_{0}$ (Fig. 10). For $\kappa=0.255$ and $\kappa=0.26$ the $\pi$ meson mass scales corresponding to the $\mathrm{PCAC}$ relation $\left(a m_{\pi}\right)^{2} \propto a m_{0}$. For larger $\kappa$, especally for those $\kappa$ between the transitions, deviations from the PCAC relation can be observed. The data for $\kappa=0.27$ on the $12^{4}$ lattice might suggest a straight line joining the origin and the two smaller points. But the data for $a m_{0}=0.01$ on the $16^{4}$ lattice indicate that we have to be aware of finite size effects and the data on the larger lattice favor a violated PCAC relation. These ambiguities in the extrapolations are not uncommon in the numerical studies of phase transition. We conclude that also the PCAC analysis does not reliably locate $\kappa_{c}$ of the chiral transition at $\beta=0.848$.

Because of the unusual behavior of the chiral condenstate the equation of state also fails. So we cannot decide from this data if the Higgs and chiral transition meet at the endpoint, as observed in the dynamical theory $[23,26]$. 


\subsection{Equation of State}

The results of the susceptibility ratio pose at least two questions: 1) is the critical behaviour at $\beta=0.30$ really different from the one at $\beta=0.00$ ? 2) if yes, and the data at $\beta=0.30$ are described by a power law scaling, what are the other critical exponents? - in particular, we are interested in the relative position of percolation and chiral transition, and in their correlation length exponents $\nu$.

The standard way to address these questions is to exploit the chiral equations of state (EOS). First we briefly discuss the results at $\beta=0.00$, which we shall use as a term of comparison, and then we will devote more time to the discussion at $\beta=0.30$.

The EOS framework is borrowed from ancient studies of ferromagnetic transitions, and, as such, uses data in the thermodynamic limit. Happily, this approach requires that we simulate the model within its scaling window but not necessarily directly at the critical point. Consequently it is possible to work with data free from finite volume effects (which won't be possible at the critical point where a finite size scaling analysis would be mandatory). Both the selection of the scaling window, and the control over finite size effects are discussed below.

Recall the possible critical behaviour, and the relative equations of state. If scaling holds, the data are described by a 'standard' equation of state

$$
a m_{0}=\langle\bar{\chi} \chi\rangle^{\delta} f\left(t /\langle\bar{\chi} \chi\rangle^{1 / \beta_{\chi}}\right),
$$

where $t$ is the reduced coupling (in our case $t=\kappa-\kappa_{c}$ ) and whose first order approximation - used in actual fits - reads

$$
a m_{0}=a^{P}\left(\kappa-\kappa_{c}\right)\langle\bar{\chi} \chi\rangle^{\delta-1 / \beta_{\chi}}+b^{P}\langle\bar{\chi} \chi\rangle^{\delta} .
$$

If the theory is trivial, the most natural candidate to describe the data is an equation of state à la Nambu-Jona-Lasinio. We will use the following form, which is motivated by the leading term of the $1 / \mathrm{N}$ expansion [42]:

$$
a m_{0}=a^{N J L}\left(\kappa-\kappa_{c}\right)\langle\bar{\chi} \chi\rangle+b^{N J L}\langle\bar{\chi} \chi\rangle^{3}(\log \langle\bar{\chi} \chi\rangle / s)
$$

and we will just comment on its possible generalizations [46,47] (in practical analysis $\log \langle\bar{\chi} \chi\rangle / s$ will be replaced by $\log \langle\bar{\chi} \chi\rangle+c)$.

In many cases we used two different fitting procedures. 


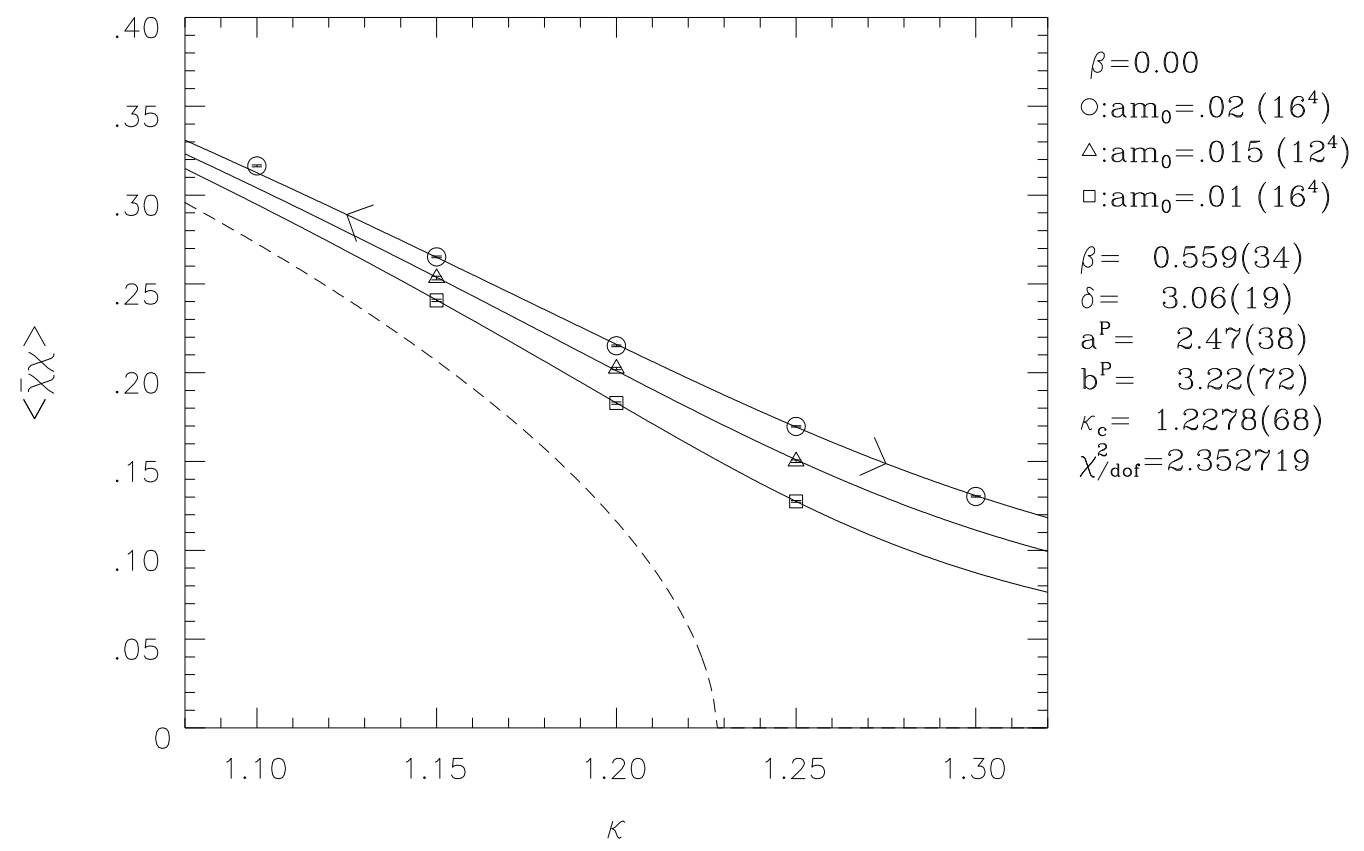

Fig. 11. Results of the power law fits described in the text at $\beta=0.00$. The arrows indicate the fitted interval.

For the first one we numerically invert eq. $(22)$ for $\langle\bar{\chi} \chi\rangle$ and then make a minimum $\chi^{2}$ fit for the measured chiral condensate, taking into account the errors. We label the results obtained with this procedure [F1].

The second one [F2] is a least squares procedure which minimizes $\left(a m_{0}-\right.$ $\left.a m(\langle\bar{\chi} \chi\rangle))^{2}, a m(\langle\bar{\chi} \chi\rangle)\right)^{2}$ being the r.h.s. of either eq. (22) or eq. (23). We then compute the errors by jack-knifing the results obtained by discarding one point at a time. The quality of the fit is estimated by $Q=\left(m_{0}-\right.$ $m(\langle\bar{\chi} \chi\rangle))^{2} /\left(\Delta\left(m(\langle\bar{\chi} \chi\rangle)^{2}\right) N_{\text {points }}\right)$. For a good fit we expect $Q$ close to one.

\section{$6.2 .1 \beta=0.00$}

As mentioned above, we expect that at $\beta=0$ the model reduces to a lattice NJL model. Recent numerical studies of four dimensional NJL models include [42-44].

At $\beta=0.00$ we restricted ourselves to $\kappa=(1.15,1.20,1.25)$. A power law fit with 5 free parameters which uses the data at bare masses .02 and .01 from the $16^{4}$ lattice, the data at .015 from the $12^{4}$ lattice is shown in Figure 11. The quality of the fit [F1] is nice, the exponents $\delta=3.06(19), \beta_{\chi}=0.56(4)$ are all consistent with mean field theory. The critical coupling $\kappa_{c}$ is 1.228(7).

We have then included logarithmic corrections, initially without scale (the parameter $s=1$ in the EOS). We used data from the $12^{4}$ lattice, and the 
procedure $[\mathrm{F} 2]$. In the same interval we obtain $\kappa_{c}=1.259(4)$. Adding one further point at $\kappa=1.30, a m_{0}=.02$ moves the central values to $\kappa_{c}=1.258$. The inclusion of a free scale parameter in the fits moves the critical point to $\kappa_{c}=1.248$, closer to the power law results. We have checked that we can discard the points with bare mass .02 without altering the results. We can also constrain the exponents to their mean field values without appreciably degrading the quality of the fits - that corresponds to 'cancelling' the logs in the NJL equation of state. $\kappa_{c}$ is then $1.224(8)$.

In conclusion, the data at $\beta=0.00$ are well described, as expected, by a mean field critical behaviour, with $\kappa_{c}$ in the range $1.22-1.26$, in agreement with the ratio analysis. This range of critical couplings is well separated from that of monopole percolation as can be seen from Table 1 and Fig. 5. Logarithmic corrections, if any, are small, their inclusion improves the quality of the fits, but does not alter $\kappa_{c}$ nor do they allow fits on a wider interval. These critical couplings are significantly different from those of the percolation transition.

\subsection{2 $\beta=0.30$}

Our first task is to select a reliable data sample for our fits. From the results for $R_{\pi}$, we know that masses larger than .02 are too heavy. On the other hand, small masses, which require large lattices, are only available for three $\kappa^{\prime} s$. All in all, our candidate window for fits narrows down to the nine points at $\kappa=.85, .87, .90$ - we occasionally checked the stability of our results by including the two $a m_{0}=.02$ points at $\kappa=.80$ and .95 but we did not venture any further. By contrasting data in this range obtained on a $8^{4}, 12^{4}$, and a $16^{4}$ lattice, we checked that finite volume effects are already small on a $8^{4}$ lattice, and that the difference between results on a $12^{4}$ and on a $16^{4}$ lattice is statistically not significant (the worst case is $a m_{0}=0.005$ were the data are separated by two standard deviations). We used the data on the $16^{4}$ lattice for our fits.

The results from [F1] applied to the power law form are shown in Figure 12. The stability of these results was checked by performing the [F2] fits on the same range of parameters. The results are shown in Table 2, first line. We get $\kappa_{c}=.874(6), \delta=2.12(15)$, and $\beta_{\chi}=0.88(5)$. (These exponents are very close to those of quenched, non-compact QED: $\delta=2.12(1), \beta_{\chi}=0.86(3), \gamma=1.0$ $[45,35]$.) Assuming scaling, these critical exponents give $\nu=.695$. We have verified that the fit is stable against the inclusion of the two points at $a m_{0}=$ $.02, \kappa=.80$ and .95 . Finally, since we want to contrast the position of the chiral transition with the position of the peaks of the monopole susceptibility, we have also tried fits constraining the critical $\kappa=.882$, at the the lower bound of $\kappa_{c}$ percolation. The results for the critical exponents (second line of Table 2) are compatible with the previous ones and give $\nu=.73$. The quality 


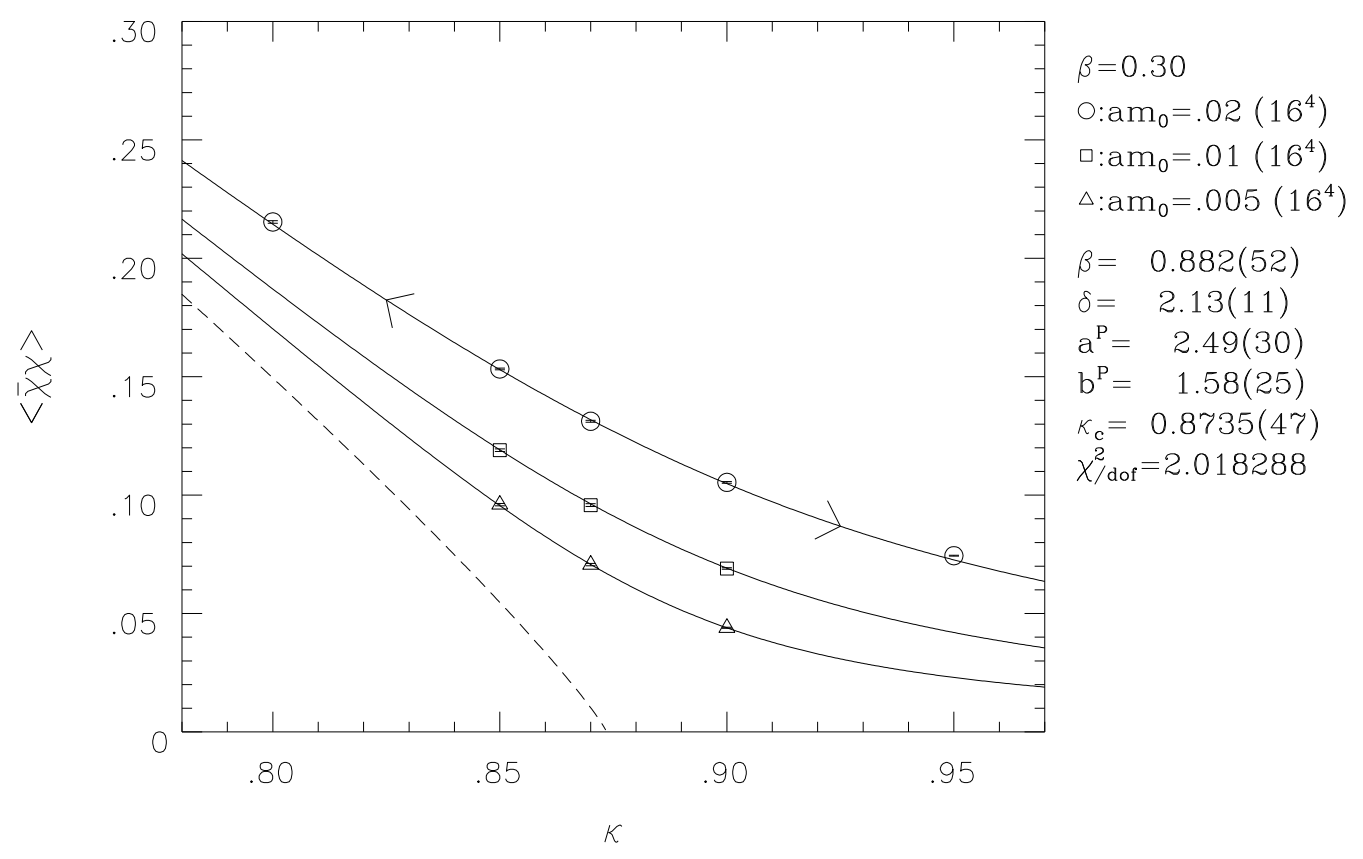

Fig. 12. As Figure 11, but $\beta=0.30$. This fit correspond to the first line in table 2 .

Table 2

Results of the powerlaw EOS (eq. (22)) fits at $\beta=0.30$.

\begin{tabular}{lllll|l}
$a^{P}$ & $b^{P}$ & $\kappa_{c}^{P}$ & $\delta$ & $\delta-1 / \beta_{\chi}$ & $\mathrm{Q}$ \\
\hline $2.54(4)$ & $1.58(36)$ & $.874(6)$ & $2.12(15)$ & $.999(68)$ & 1.068 \\
$2.19(28)$ & $1.22(7)$ & $.882[$ Fixed] & $1.94(2)$ & $.932(47)$ & 1.79 \\
$2.00(40)$ & $1.08(8)$ & $.8875[$ Fixed] & $1.83(3)$ & $.893(82)$ & 2.96
\end{tabular}

parameter Q (1.79) is still acceptable. Constraining $\kappa_{c}$ deeper into the critical interval spanned by the critical $\kappa$ for percolation further degrades the quality of the fit, which remains neverthless tolerable. We have also searched for the best exponents assessing by eye the quality of the scaling plots built following eq. (21). The best plot picks $\kappa_{c}=.868, \delta=2.26, \beta_{\chi}=.8$, giving $\nu=.65$.

We have also tried to fit the data with a logarithmic improved mean field equation. The results of the fits are displayed in Table 3. (Note, that this fit has less free parameters.) We see that the only fit of quality comparable to the power law fits requires a variable scale. In this case, the critical coupling is smaller than that from the power law fits: $\kappa_{c}=.866(2)$ to be contrasted with $\kappa_{c}=.874(6)$. The shift in the critical coupling with respect to the power law results can also be appreciated by performing a constrained fit with $\kappa_{c}=.882$, which fails completely (third line of Table $3, \mathrm{Q}=83.26$ ): this definitively shows that a NJL critical behaviour cannot be associated with the percolation of magnetic monopoles. 
Table 3

Results of the logarithmic EOS (eq. (23)) fits at $\beta=0.30$

\begin{tabular}{llll|l}
$a^{N J L}$ & $b^{N J L}$ & $\kappa_{c}^{N J L}$ & $c$ & $\mathrm{Q}$ \\
\hline $2.46(6)$ & $-3.68(16)$ & $.861(1)$ & $0[$ Fixed] & 6.94 \\
$2.54(5)$ & $-6.12(84)$ & $.866(2)$ & $.69(14)$ & 1.18 \\
$2.81(19)$ & $-13.88(2.01)$ & $.882[$ Fixed] & $1.23(13)$ & 83.26
\end{tabular}

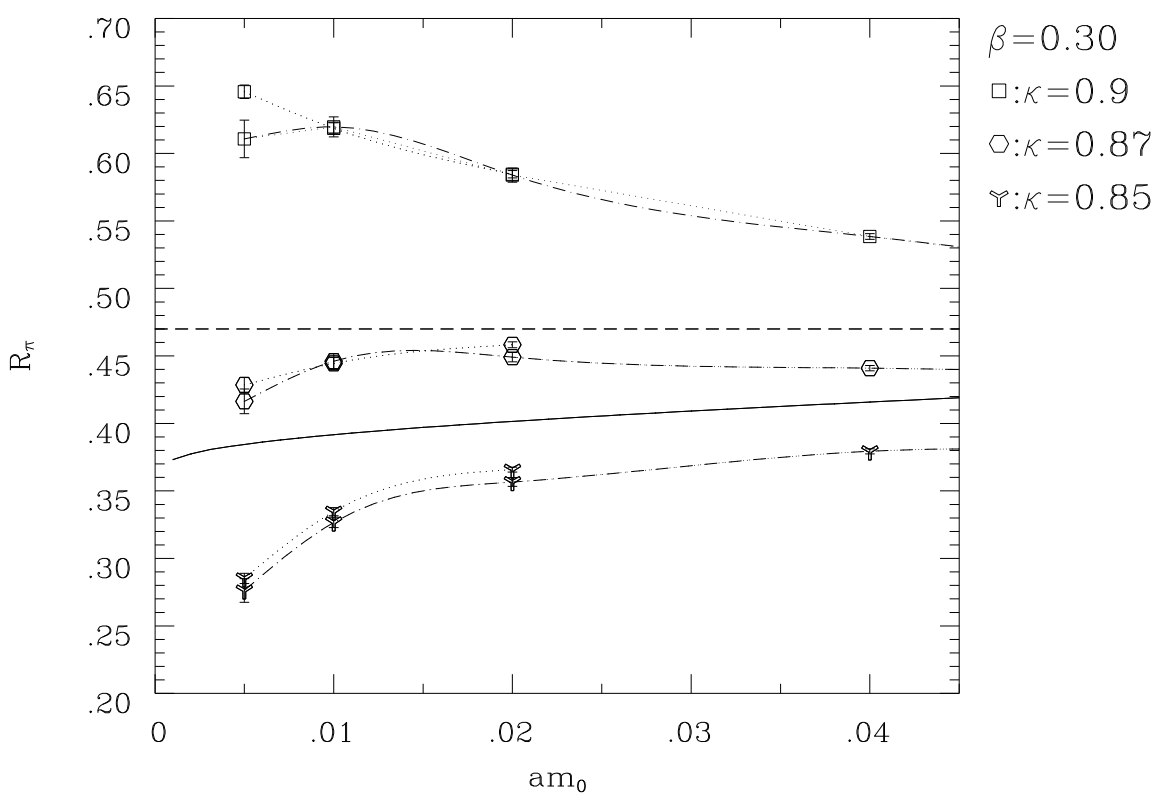

Fig. 13. $R_{\pi}$ in the critical region. Dotted is for the $16^{4}$ lattice, dot-dash for the $12^{4}$. The dashed line is $R_{\pi}\left(\kappa_{c}\right)=1 / \delta$ from the power law fit Table 2 , the solid line is $R_{\pi}\left(\kappa_{c}\right)$ from the logarithmic fit, first line Table 3.

We can also study the sensitivity of the position of the critical point to the equation of state by inspecting $R_{\pi}$. This gives a clearer indication of the role played by the precise location of the critical point in determining the critical scaling. In Fig. 13 we show the results for $R_{\pi}$ in the critical region constrasted with $1 / \delta$ (solid line) from our best power law fit, and with $R_{\pi}\left(\kappa_{c}\right)$ from the logarithmic fit without scale. We see that the $R_{\pi}$ 's corresponding to the two hypotheses (logarithms and power law) fall in the $\kappa$ interval predicted by the fits - between .85 and .87 for $\log$ fits $\left(\kappa_{c} \simeq .86\right)$, and between .87 and .90 for power law $\left(\kappa_{c} \simeq .88\right)$.

Summarizing: If the correct results (i. e. those describing the physics of the chiral limit on infinite volumes) are given by the logarithmic fits, second line Table 3, the percolation transition and the chiral transition are distinct. In this case we have not learned anything about a possible role of magnetic monopoles in the existence of strongly coupled theories. Simply, the chiral 
transition would be trivial and magnetic monopoles would have nothing to do with it, as it is for $\beta=0.00$ and probably also for $\beta=0.848$.

If the correct results are given by power law fits, Table 2, the chiral transition coincides within errors with the percolation transition. The critical exponent $\nu$ for the chiral transition is in the range $.65-.73$, to be contrasted with

$\nu_{\text {perc }}=.61(4)$, as we determined it in section 5. Taking into account the possibility of further corrections, the exponents $\nu$ for the two transitions are compatible and $\simeq 0.65$.

Note again these results differ from the ones in the full model where the critical behavior along the line NE seems the same as the one of the trivial NJL point. It is not in the scope of the present paper to investigate the relationship between the quenched and full model, but this is certainly an interesting subject for further investigations.

Finally, we might wonder about the sensitivity of the quality of the logarithmic fits and/or the value of the critical coupling to further corrections. Typical corrections include powers of the logarithms. Although such corrections are not there, up to $\mathrm{O}\left(1 / N^{2}\right)$, they cannot be excluded in general. Our model, however, could be in the Yukawa universality class $[46,47]$. In this case, one can conceive of sizeable variation, even in sign, of the exponents and the simplest parametrization of such crossover behavior would suggest that the powers in the logs should be kept free. It cannot be excluded that more general logarithmic equations of state fit the data well, and certainly this would make the study of the interrelation between the chiral transition and monopole percolation more subtle.

\section{Summary}

We have investigated the topological excitations in the phase diagram of scalar QED. We confirmed with great precision the picture suggested by [28] and see clear signals for first order phase transitions in the topological observables.

We have investigated in detail chiral transition and monopole percolation for three points:

- $\beta=0.00$ : The chiral transition is described by mean field exponents, possibly with small (logarthmic) corrections. The percolation transition is well separated from the chiral transition.

- $\beta=0.30$ : Two possibilities are consistent with the data: $1^{\text {st }}$ a situation analogous to that at $\beta=0.00$, but with large logarithmic corrections; $2^{\text {nd }}$ percolation and chiral transition coincident, with the same critical exponent 
$\nu$.

- $\beta=0.848$ : The only clear statement here is that the Higgs transition and monopole percolation are distinct. We have not been able to measure with confidence the chiral transition, which has proven hard to study with conventional numerical methods.

Since the scalar field increases the order in the gauge fields and favours chiral symmetry, separate chiral and monopole percolation transitions are not unexpected: it might well be that monopoles percolate in the symmetric phase, but their tendency to break chiral symmetry is overcome by the ordering effects of the scalar fields. This seems to be the case at $\beta=0.00$, and it is also possible at $\beta=0.30$ (if the first possibility is realized). In this case chiral transition is most probably mean field like along the whole line NE (without the point E), as it seems to be in the full model. Moreover, the chiral transition is separated from the percolation transition: when this is the case monopoles should be irrelevant to the dynamics of the chiral transition in the large volume, continuum limit. Except for the point E (we reiterate that we don't have any conclusion on the relative position of monopoles and chiral transition at the point $\mathrm{E}$ ) this goes hand in hand with the transition being trivial. Still, the behaviour at $\beta=0.30$ remains different from that at $\beta=0.00$, and and it would be interesting to find a coherent scenario accomodating these observations.

The scenario of [5] requires instead a chiral transition coincident with the percolation transition, and sharing the same correlation length exponent. When this occurs, the dynamics of the chiral transition should inherit the characteristics of that of the magnetic monopoles, which survive the non-trivial continuum limit. This could be the case at $\beta=0.30$. The scenario of [5] receives then some support from our investigation.

Important questions concern the relevance of these results for the full model, and, in general, the sensitivity to the number of flavours. If the scenario of [5] is realized, the situation is different from the one observed in the full model: If this is the case, fermion screening plays an important role in this model as it presumably does in the gauged Nambu Jona Lasinio model: there the ladder (quenched) approximation predicts a non-mean field scenario [48,49], while the full model is presumably trivial[50]. These observations do not detract from the illustrative value of our results at $\beta=0.30$, but, of course, prevent us from extending any of our conclusions to the full model at this stage of our investigation. If the full and quenched models really have different critical behavior along the line NE, then we are challenged to understand something unexpected and special about fermionic screening in this model. In the present quenched model, the dynamical scalar field in the configurations completely screens the electric charge, producing a vanishing renormalized gauge coupling in this sector of the model. If the fermions were also treated dynamically, they would add nothing qualitatively new in terms of charge screening. Therefore, 
the fermion-monopole interaction would have to have some essential ingredient which renders the monopoles ineffective in driving chiral symmetry breaking in the full model. Simulations of non-compact QED with the number of flavors $N_{f}$ varying from 2 to 32 , do not show such an effect - there chiral symmetry breaking and monopole percolation were coincident, within ample errorbars, for all $N_{f}$ [5]. Better simulations of the full and quenched models with smaller bare fermion masses and larger lattices are needed now.

We should define better numerical strategies so to show more clearly the correlation, or lack thereof, between chiral and monopole observables. It is however evident from our data, that for $\beta=0.00$, when the chiral and monopole transitions are clearly distinct, the chiral transition is clearly trivial and is not correlated with the percolation of monopoles. When the transitions grow closer for $\beta=0.30$, so do the correlation length exponents $\nu$ (or the effective critical exponent $\nu$ in the case of logarithmic triviality). The transitions may be coincident, with the same critical exponent $\nu$.

\section{Acknowledgements}

We thank J. Jersák for stimulating discussions, for reading the manuscript and for usuful comments. This study used the codes developed for the $\chi U \phi_{4}$ project for the field dynamics and the chiral observables, and the Hands-Wensley routine for the magnetic monopole meassurements. Most of the computations have been performed on the CRAY-YMP and T90 of HLRZ Jülich, for some large lattices and small masses we used the CRAY-C90's of PSC and NERSC. WF thanks HLRZ for hospitality. JBK thanks the Pittsburgh Supercomputer Center and the National Energy Research Supercomputer Center for access to their facilities. MPL thanks the Physics Department of the University of Bielefeld for its hospitality. This work was supported by: DFG, the National Science Foundation, nsf-phy9605199, the U.S. Department of Energy (D.O.E.) under cooperative research agreement \#DF-FC02-94ER40818, and by Nato grant no. CRG 950896.

\section{References}

[1] A. K. De and J. Jersák, in A. J. Buras and M. Lindner, eds., Heavy Flavors, p. 732 (World Scientific, Singapore, 1992).

[2] R. E. Shrock, in M. Creutz, ed., Quantum fields on the computer, p. 150 (World Scientific, Singapore, 1992).

[3] M. B. Einhorn and R. Savit, Phys. Rev. D17 (1978) 2583; D19 (1979) 1198. 
[4] T. Banks, R. Myerson, and J. Kogut, Nucl. Phys. B129 (1977) 493.

[5] J. B. Kogut and K. C. Wang, Phys. Rev. D53 (1996) 1513 and references therein.

[6] A. Kocić, J. B. Kogut, and S. J. Hands, Phys. Lett. 289B (1992) 400.

[7] S. J. Hands, A. Kocić, J. B. Kogut, R. L. Renken, D. K. Sinclair, and K. C. Wang, Nucl. Phys. B413 (1994) 503.

[8] A. Kocić, J. B. Kogut, and K. C. Wang, Nucl. Phys. B398 (1993) 405.

[9] A dissenting view is offered by M. Göckeler et al., Nucl. Phys. B371 (1992) 713.

[10] J. Jersák, C. B. Lang, and T. Neuhaus, Phys. Rev. Lett. 77 (1996) 1933.

[11] J. Jersák, C. B. Lang, and T. Neuhaus, Phys. Rev. D54 (1996) 6909.

[12] J. Cox, W. Franzki, J. Jersák, C. B. Lang, T. Neuhaus, and P. W. Stephenson, Gauge-ball spectrum of the four-dimensional pure $U(1)$ gauge theory, HLRZ 84/96, hep-lat/9701005, to be published in Nucl. Phys. B.

[13] J. Cox, W. Franzki, J. Jersák, C. B. Lang, and T. Neuhaus, Strongly coupled compact lattice QED with staggered fermions, PITHA 97/22, HLRZ 19/97, heplat/9705043.

[14] I.-H. Lee and R. E. Shrock, Phys. Rev. Lett. 59 (1987) 14.

[15] I.-H. Lee and J. Shigemitsu, Phys. Lett. 178B (1986) 93.

[16] For recent reviews see A. Kocić Nucl. Phys. B (Proc. Suppl.) 34 (1994) 123, V. Azcoiti Nucl. Phys. B (Proc. Suppl.) 53 (1997) 148.

[17] M. Baig, H. Fort, J. B. Kogut, S. Kim, and D. K. Sinclair, Phys. Rev. D48 (1993) 2385.

[18] M. Baig, H. Fort, J. B. Kogut, and S. Kim, Phys. Rev. D51 (1995) 5216.

[19] J. L. Alonso et al., Nucl. Phys. B405 (1993) 574.

[20] L. F. Abbott and E. Farhi, Phys. Lett. 101B (1981) 69.

[21] E. Dagotto and J. Kogut, Phys. Lett. 208B (1988) 475.

[22] C. Frick and J. Jersák, Phys. Rev. D52 (1995) 340.

[23] W. Franzki, C. Frick, J. Jersák, and X. Q. Luo, Nucl. Phys. B453 (1995) 355.

[24] W. Franzki and J. Jersák, Nucl. Phys. B (Proc. Suppl.) 47 (1996) 663.

[25] W. Franzki, Nucl. Phys. B (Proc. Suppl.) 53 (1997) 702.

[26] W. Franzki and J. Jersák, Dynamical fermion mass generation at a tricritical point in strongly coupled $U(1)$ lattice gauge theory, in preparation. 
[27] W. Franzki, Dynamische Erzeugung von Fermionmassen in stark gekoppelten Eichtheorien auf dem Gitter, Dissertation (RWTH Aachen, 1997).

[28] J. Ranft, J. Kripfganz, and G. Ranft, Phys. Rev. D28 (1983) 360.

[29] A. M. Horowitz, Phys. Lett. 219B (1989) 329.

[30] H. B. Nielsen and P. Olesen, Nucl. Phys. B61 (1973) 45.

[31] T. A. DeGrand and D. Toussaint, Phys. Rev. D22 (1980) 2478.

[32] S. Hands and R. Wensley, Phys. Rev. Lett. 63 (1989) 2169.

[33] E. Dagotto, J. B. Kogut, and A. Kocic, Phys. Rev. D43 (1991) R1763.

[34] A. Kocić, J. B. Kogut, and M.-P. Lombardo, Nucl. Phys. B398 (1993) 376.

[35] M.-P. Lombardo, A. Kocić, and J. B. Kogut, More on strongly coupled quenched QED, hep-lat/9411051.

[36] D. Staufer, Phys. Rep. 54 (1979) 1.

[37] G. W. Kilcup and S. R. Sharpe, Nucl. Phys. B283 (1987) 493.

[38] We thank J.-F. Lagaë for discussions on this point.

[39] M. Baig, H. Fort, and J. B. Kogut, Phys. Rev. D50 (1994) 5920.

[40] We thank G. Parisi for discussions on percolation.

[41] H. G. Ballesteros, L. A. Fernandez, V. Martin-Mayor, A. M. Sudupe, G. Parisi, and J. Ruiz-Lorenzo, Measures of critical exponents in the four-dimensional site percolation, preprint hep-lat/9612024.

[42] S. Kim, A. Kocić, and J. Kogut, Nucl.Phys. B429 (1994) 407 and references therein.

[43] A. Ali Khan, M. Göckeler, R. Horsley, P. E. L. Rakow, G. Schierholz, and H. Stüben, Phys. Rev. D51 (1995) 3751.

[44] S. Hands and J. B. Kogut, Logarithmic corrections to the equation of state in the $S U($ Q) $\times S U($ Q) $N J L$ model, hep-lat/9705015.

[45] A. Kocic, J. B. Kogut, M.-P. Lombardo, and K. C. Wang, Nucl. Phys. B397 (1993) 451.

[46] A. Hasenfratz, P. Hasenfratz, K. Jansen, J. Kuti, and Y. Shen, Nucl. Phys. B365 (1991) 79.

[47] J. Zinn-Justin, Nucl. Phys. B367 (1991) 105.

[48] W. A. Bardeen, S. T. Love, and V. A. Miransky, Phys. Rev. D42 (1990) 3514.

[49] A. Kocic, S. Hands, J. B. Kogut, and E. Dagotto, Nucl. Phys. B347 (1990) 217.

[50] S. Kim, J. B. Kogut, and M.-P. Lombardo, in preparation. 